\title{
The in vivo RNA structurome of the malaria parasite Plasmodium falciparum, a protozoan with an A/T-rich transcriptome
}

\author{
Dumetz, $\mathrm{F}^{1 \uparrow \pm}$, Enright, $\mathrm{AJ}^{1 \uparrow}$, Zhao, $\mathrm{J}^{2}$, Kwok $\mathrm{CK}^{2-3^{\star}}$, Merrick, $\mathrm{CJ}^{1^{\star}}$
}

\section{Affiliations:}

${ }^{1}$ Department of Pathology, University of Cambridge, Cambridge CB2 1QP, UK

${ }^{2}$ Department of Chemistry and State Key Laboratory of Marine Pollution, City University of Hong Kong, Kowloon Tong, Hong Kong SAR, China

${ }^{3}$ Shenzhen Research Institute of City University of Hong Kong, Shenzhen, China

${ }^{\uparrow}$ Authors contributed equally to this work

${ }^{ \pm}$Current affiliation: Institute for Genome Sciences, University of Maryland School of Medicine, Baltimore, 21201, Maryland, USA

${ }^{*}$ Corresponding authors:

c.merrick@keele.ac.uk (CJM)

ckkwok42@cityu.edu.hk (CKK)

\section{Keywords:}

Malaria, Plasmodium falciparum, RNA, Structurome, NAI, Structure-seq.

\begin{abstract}
Plasmodium falciparum, a protozoan parasite and causative agent of human malaria, has one of the most A/T-biased genomes sequenced to date. This may give the genome and the transcriptome unusual structural features. Recent progress in sequencing techniques has made it possible to study the secondary structures of RNA molecules at the transcriptomic level. Accordingly, in this study we produced the first in vivo RNA structurome of a protozoan parasite, and the first of a highly $\mathrm{A} / \mathrm{U}$-biased transcriptome. We showed that it is possible to probe the secondary structure of $P$. falciparum RNA molecules in vivo using two different chemical probes, and obtained structures for more than half of all transcripts in the transcriptome. These showed greater stability (lower free energy) than the same structures modelled in silico, and structural features appeared to influence translation efficiency and RNA decay. Finally, we compared the $P$. falciparum RNA structurome with the predicted RNA structurome of an A/T-balanced species, $P$. knowlesi, finding a bias towards lower overall transcript stability and more hairpins and multi-stem loops in $P$. falciparum. This first protozoan RNA structurome will provide a basis for similar studies in other protozoans and also in other unusual genomes.
\end{abstract}

\section{Introduction}

RNA molecules, composed of single strands of four different ribonucleotides, do not adopt a single canonical structure like the double helix of DNA; instead they can fold into complex secondary structures made of loops, hairpins and bulges (1). They serve a variety of roles in the cell $(2,3)$, including structural roles carried out by 
transfer and ribosomal RNAs (tRNAs, rRNAs) and protein coding roles for messenger RNAs (mRNAs). These roles are facilitated by the ability of RNA molecules to adopt, and transition between, highly specific secondary structures (4, $5)$. Different secondary structures can impact on the location of the RNA molecules, their metabolism and stability (6), their interaction with RNA binding proteins (7), their function and their regulation of protein expression. Two outstanding examples of this are the function-related shape of tRNA (8) and the translation expression controlling element, the Riboswitch (9).

One of the common strategies to investigate RNA structures in vivo is

Selective 2'-Hydroxyl Acylation analysed by Primer Extension (SHAPE) (10). SHAPE uses chemicals that modify the 2' hydroxyl group of flexible nucleotides, in which the 2' O-adduct will inhibit reverse transcription during cDNA synthesis (11). The pattern of stalling is then detected on a sequencing gel, which determines the identity of all unpaired/flexible bases in the RNA, and hence allows its secondary structure to be inferred. The technicalities of SHAPE only permit the analysis of one transcript at the time, requiring a high amount of starting RNA and potentially more than one sequencing gel to analyse larger transcripts. All these technical limitations rendered impossible the transcriptome-wide study of RNA structures, which is referred to as a structurome (12). However, the advent of Next Generation Sequencing (NGS) and bio-informatics revolutionised the field of RNA structure and within the past decade, Structure-seq (13), and many other related methods $(14,15)$, were developed.

To date, only a few in vivo structuromes are available. Many viruses have been studied, probably due to their small number of RNA molecules, such as HIV (16), DENV and ZIKV $(17,18)$, influenza (19) and more recently SARS-CoV-2 (2024). Regarding eukaryotes, structuromes were established for model organisms like the plants Arabidopsis thaliana (13) and Oryza sativa (rice) (25), the yeast Saccharomyces cerevisiae (26), the bacterium Escherichia coli (27) and amongst mammals, mouse embryonic stem cells $(27,28)$ and human cell lines $(6,26,27,29)$. These have revealed fundamental conserved features across the landscape of RNA structures.

In this paper we have established the first in vivo structurome of a protozoan, Plasmodium falciparum. $P$. falciparum is one of the etiological agents of malaria, the most widely lethal human parasitic disease in the world (30). Little is known about RNA structures in $P$. falciparum, yet the parasites offer many opportunities to understand the roles of RNA structures in eukaryotic cells. Firstly, the natural life cycle of the parasite oscillates from a warm-blooded host to an insect vector, meaning that the same organism is exposed to very different temperatures and metabolic environments. This allows the study in natural conditions of the effect of the environment and temperature upon the RNA structurome. Indeed, it has been shown that heat stress can affect the structuromes of plants (31) and bacteria (32, 33), which can likewise experience temperature extremes in nature. Furthermore, one intriguing piece of evidence concerning rRNAs suggests that temperatureresponsive RNA structures are also important in Plasmodium life cycles.

Plasmodium does not encode rRNAs in conventional tandem arrays, but rather in several isolated units which are transcribed independently in either mosquito stages (S-type) or mammalian stages (A-type) $(34,35)$. rRNA transcription apparently switches in response to differential temperature and nutrient availability in the two hosts $(36,37)$. The two types of rRNA were predicted, based on their sequences, to have different structures (38) and they do perform differently when complemented into $S$. cerevisiae (39), so there is clear potential for them to influence translation in 
insects versus humans. Thus, Plasmodium may have evolved variant RNA structures as a key element in its life cycle transitions.

Secondly, while all the previously cited organisms have a more balanced ratio of $\mathrm{A} / \mathrm{T}$ and $\mathrm{G} / \mathrm{C}$ in their genomes, $P$. falciparum is $80.6 \% \mathrm{~A} / \mathrm{T}$-rich (40): one of the most biased genome compositions ever to be sequenced. This offers a critical point of view when studying genome evolution, and it may well lead to unusual RNA structures.

This first ever in vivo RNA structurome of $P$. falciparum intends to investigate the relationship between RNA shapes and genome composition, as well as transcription efficiency, in the asexual erythrocytic cycle of this important human parasite.

\section{Material and methods}

\section{Parasite culture}

Plasmodium falciparum 3D7, obtained from the Malaria Research and Reference Reagent Resource Center (MR4), was cultured in O+ human erythrocytes at 4\% haematocrit in RPMI 1640 supplemented with $0.25 \%$ albumax (Invitrogen), 5\% heatinactivated human serum and $0.25 \%$ sodium bicarbonate in gassed chambers at $1 \%$ $\mathrm{O}_{2}, 3 \% \mathrm{CO}_{2}$, and $96 \% \mathrm{~N}_{2}(41)$. Parasite count was assessed on thin blood smears stained with Hemacolor (Merck).

\section{RNA structure probing and poly $(A)$ enrichment}

An asynchronous culture was grown up to $1.2 \times 10^{10}$ parasites as previously described (41). Erythrocytes were pelleted and then incubated for 30 minutes either with $10 \mathrm{mM}$ DMS (dimethyl sulfate, Sigma) or $20 \mathrm{mM} \mathrm{NAI}$ (2-methylnicotinic acid imidazolide), at $37^{\circ} \mathrm{C}$, agitated at $200 \mathrm{rpm}$, in the same gas mixture described above. The NAI was prepared as previously described (42). The reaction was stopped with $40 \mathrm{mM}$ dithiothreitol (DTT). Parasites were extracted by erythrocyte lysis using $1 \mathrm{v}$ of $0.2 \%$ saponin in PBS and then washed 3 times in ice-cold PBS. RNA was extracted using Qiagen RNeasy plus mini kit (Qiagen) following manufacturer's instructions and then submitted to DNase treatment for 15 minutes (Qiagen). The total RNA recovered was split into samples of $5 \mu \mathrm{g}$ each and enriched in poly $(A)$ transcripts using NEBNext Poly(A) mRNA magnetic isolation module (NEB) according to manufacturer's instructions.

\section{Visualisation of 5.8S rRNA structure using RT-stalling}

RT stalling was performed using a primer targeting the 5.8S rRNA gene (PF3D7_0531800). Briefly, $1.5 \mu \mathrm{g}$ of total RNA from DMS, NAI and DMSO-treated cultures in $5.5 \mu \mathrm{l}$ nuclease-free water was mixed with $1 \mu \mathrm{l}$ of $5 \mu \mathrm{M}$ Pfa_5.8S primer labelled at the 5' end with Cy5 [Cy5]ATTTTCTGTAGGAGTACCACT (Eurofins Genomics) in $3 \mu \mathrm{l}$ of reverse transcription buffer (20 mM Tris, $\mathrm{pH} 7.5,4 \mathrm{mM} \mathrm{MgCl}, 1$ $\mathrm{mM}$ DTT, $0.5 \mathrm{mM}$ dNTPs, $150 \mathrm{mM} \mathrm{LiCl}$ in final). For the sequencing reactions $(\mathrm{A} / \mathrm{G} / \mathrm{C} / \mathrm{T})$, an extra $1 \mu \mathrm{l}$ of $10 \mathrm{mM}$ dideoxynucleoside triphosphate (Roche) was added to replace $1 \mu \mathrm{l}$ of nuclease-free water. All samples were heated at $75^{\circ} \mathrm{C}$ for 3 $\min , 35^{\circ} \mathrm{C}$ for $5 \mathrm{~min}$ and then held at $50^{\circ} \mathrm{C}$. Next, $0.5 \mu \mathrm{l}$ of Superscript III $(200 \mathrm{U} / \mu \mathrm{l})$ (Thermo Scientific) was added and reverse transcription was performed at $50^{\circ} \mathrm{C}$ for $15 \mathrm{~min}$, followed by addition of $0.5 \mu \mathrm{l}$ of $2 \mathrm{M} \mathrm{NaOH}$ at $95^{\circ} \mathrm{C}$ for $10 \mathrm{~min}$ to degrade the RNA template. After reverse transcription, $10 \mu \mathrm{l}$ of $2 x$ formamide orange $\mathrm{G}$ dye $(94 \%$ deionized formamide, $20 \mathrm{mM}$ Tris $\mathrm{pH}$ 7.5, 20 mM EDTA, orange G dye) was added 
and heated at $95^{\circ} \mathrm{C}$ for 3 min before loading into a pre-heated (at $90 \mathrm{~W}$ for $45 \mathrm{~min}$ ) $8 \%$ denaturing polyacrylamide gel. The gel was run at constant power of $90 \mathrm{~W}$ for 90 min after loading $3.2 \mu \mathrm{l}$ of each sample. Fujifilm FLA 9000 was used to scan the gel.

\section{Library preparation and sequencing details}

Random fragmentation of polyA-enriched RNA was performed with 150 ng polyAenriched RNA in fragmentation buffer $(40 \mathrm{mM}$ Tris- $\mathrm{HCl} \mathrm{pH} 8.2,100 \mathrm{mM} \mathrm{LiCl}, 30 \mathrm{mM}$ $\mathrm{MgCl}_{2}$ ) at $95^{\circ} \mathrm{C}$ for 60 s to generate average fragment size of $\sim 250 \mathrm{nt}$, and then purified with RNA Clean \& Concentrator (Zymo Research). 3' dephosphorylation reactions included $7 \mu$ l fragmented sample, $1 \mu$ l of 10x PNK buffer (NEB), $1 \mu \mathrm{l}$ of rSAP enzyme (NEB) and $1 \mu$ of PNK enzyme (NEB), carried out at $37^{\circ} \mathrm{C}$ for $30 \mathrm{~min}$. 3' adapter ligation was performed by adding 3' adapter

(5'-/5rApp/NNNNNNAGATCGGAAGAGCACACGTCTG/3SpC3/-3' with 1:5 molar ratio of RNA to 3' adapter), PEG 8000 (17.5\% final) and T4 RNA ligase 2 (NEB) in 1x T4 RNA ligase buffer at $25^{\circ} \mathrm{C}$ for $1 \mathrm{~h}$. Excess adapter was digested by adding $1 \mu \mathrm{l}$ RecJf (NEB) and $1 \mu \mathrm{l} 5^{\prime}$ deadenylase (NEB) at $30^{\circ} \mathrm{C}$ for $30 \mathrm{~min}$ and removed by RNA Clean \& Concentrator (Zymo Research). Reverse transcription reactions including the ligated RNA above, reverse primer (5'-CAGACGTGTGCTCTTCCGATCT-3' with 1:2.5 molar ratio of RNA to reverse primer), reverse transcription buffer (see RTS method above) and Superscript III (1U/ $\mu$ l final) (Thermo Scientific) were performed at $75^{\circ} \mathrm{C}$ for $3 \mathrm{~min}, 35^{\circ} \mathrm{C}$ for $5 \mathrm{~min}$, then $50^{\circ} \mathrm{C}$ for $50 \mathrm{~min}$. In this step, Superscript III should be added before the $50^{\circ} \mathrm{C}$ and after reverse transcription, $\mathrm{NaOH}(0.1 \mathrm{M}$ final) was added at $95^{\circ} \mathrm{C}$ for 10 min for RNA degradation, followed by mixing with $5 \mu \mathrm{l} 1 \mathrm{M}$ Tris- $\mathrm{HCl}$ ( $\mathrm{pH}$ 7.5) before RNA Clean \& Concentrator (Zymo Research). The purified cDNA was then ligated with 5 ' adapter

(5'/5Phos/AGATCGGAAGAGCGTCGTGTAGCTCTTCCGATCTN10/3SpC3/-3' with 1:20 molar ratio of RNA to 5'adapter) using Quick Ligation Kit (NEB) at $37^{\circ} \mathrm{C}$ overnight. Ligated reaction mixture was heated to $95^{\circ} \mathrm{C}$ for 10 min for inactivation and mixed with 1 volume of $2 x$ formamide orange $G$ dye before purifying with $10 \%$ denaturing urea-TBE acrylamide gel (Thermo Scientific) at $300 \mathrm{~V}$ for $20 \mathrm{~min}$. The size of 100-400 nt was sliced, crushed and soaked in 1x TEN 250 buffer (1x TE pH 7.4, $0.25 \mathrm{M} \mathrm{NaCl}$ ) with incubation at $80^{\circ} \mathrm{C}$ for 30 min with $1300 \mathrm{rpm}$ shaking, and then purified with RNA Clean \& Concentrator (Zymo Research). The purified ssDNA was mixed with forward primer (5'-

AATGATACGGCGACCACCGAGATCTACACTCTTTCCCTACACGACGCTCTTCCG ATCT-3', $0.5 \mu \mathrm{M}$ final) and reverse primer with different indexes (5'-

CAAGCAGAAGACGGCATACGAGAT-(6 nt index seq)-

GTGACTGGAGTTCAGACGTGTGCTCTTCCGATCT-3', $0.5 \mu \mathrm{M}$ final) for PCR amplification by using KAPA HiFi HotStart ReadyMix (KAPA Biosystems). The PCR program included $95^{\circ} \mathrm{C}$ for $3 \mathrm{~min}, 16$ cycles of 3 steps $\left(98^{\circ} \mathrm{C}: 20 \mathrm{~s}, 68^{\circ} \mathrm{C}\right.$ : $15 \mathrm{~s}, 72^{\circ} \mathrm{C}$ : $40 \mathrm{~s}), 72^{\circ} \mathrm{C}$ for $90 \mathrm{~s}$, then cooled to $4^{\circ} \mathrm{C}$ for size selection. PCR samples were resolved on a $1.8 \%$ TAE agarose gel at $120 \mathrm{~V}$ for $55 \mathrm{~min}$. The size of 150-400 nt was cut and recovered by Zymo DNA agarose gel extraction kit (Zymo Research). DNA libraries were quantified, pooled and sequenced on the Illumina Hiseq System in 150 bp paired-end (PE) configuration. A more detailed protocol is described in (43).

\section{Structurome analysis}

Forward and reverse reads from each sample were cleaned of adapter sequence contamination using reaper (v15-065) from the kraken package (44). Paired reads were then aligned to an indexed reference transcriptome (PlasmoDB- 
45_Pfalciparum3D7) using Bowtie2 (v2.4.2) in paired-end mode and converted to SAM and sorted BAM files (45). Reverse transcription stops were assessed using StructureFold2 for each individual sample (46). Coverage and overlap of RT stop data were then computed along with cross-replicate coverage and overlap and stop correlation was assessed among all samples via StructureFold2. These data were normalised and structural reactivity data generated for each transcript with enough supporting data across samples using StructureFold2. These reactivities were supplied to RNAStructure for experimental-reactivity-constrained folding (47). Resulting structures were then assessed for structural features using Forgi from the ViennaRNA package (48). Unconstrained folding of every transcript was also performed using RNAStructure with the same parameters and assessed similarly to generate a cohort of unconstrained, purely in-silico-folded transcripts. Parameters for the number of reads and mapping efficiency for each library are shown (Table 1).

\begin{tabular}{|l|r|r|r|r|}
\hline \multicolumn{1}{|c|}{ Sample } & \multicolumn{1}{c|}{$\begin{array}{c}\text { Number of } \\
\text { Read Pairs }\end{array}$} & $\begin{array}{c}\text { Yield } \\
\text { (Gigabases) }\end{array}$ & \multicolumn{1}{c|}{$\begin{array}{c}\text { Mapped } \\
\text { Reads }\end{array}$} & $\begin{array}{c}\text { Mapped } \\
\text { Reads (\%) }\end{array}$ \\
\hline Total-RNA-DMS-3 & $148,865,508$ & 44.6597 & $108,912,838$ & $73.1 \%$ \\
\hline Total-RNA-DMSO-3 & $163,681,632$ & 49.1045 & $115,215,035$ & $70.3 \%$ \\
\hline Total-RNA-NAI-3 & $192,424,134$ & 57.7272 & $144,128,728$ & $74.9 \%$ \\
\hline Total-RNA-DMS-2 & $166,742,223$ & 50.0227 & $117,106,285$ & $70.2 \%$ \\
\hline Total-RNA-DMSO-2 & $186,503,272$ & 55.951 & $128,887,798$ & $69.1 \%$ \\
\hline Total-RNA-NAI-2 & $145,601,085$ & 43.6803 & $108,451,225$ & $74.5 \%$ \\
\hline
\end{tabular}

Table 1: Sequencing parameters for each NGS library generated in this study.

\section{Functional dataset analysis}

Microarray datasets from parasites exposed to hyperoxia were extracted from (49), and the chloroquine-exposed dataset was extracted from (50). Lists of genes were updated to the current genome annotation. Ribosome profiling data were obtained from (51) and translational efficiency was calculated as ribosome density per messenger RNA. The RNA decay dataset was extracted from (52) at 10hpi for rings, 24hpi for early trophozoites, 30hpi for late trophozoites and 46hpi for schizonts. Structural parameters per transcript (numbers of nucleotides involved in stems, hairpins, multi-stem loop and bulges) were normalized to transcript length before comparing these structural parameters with the translational efficiency of each gene.

\section{Results}

\section{Assessment of NAI and DMS as RNA structure probing chemicals for $\boldsymbol{P}$. falciparum inside the erythrocyte host cell.}

We have conducted here the first in vivo Structure-seq on $P$. falciparum: an organism with a $>80 \%$ AT-rich genome, and one that lives entirely inside another host cell. It was therefore necessary to test and optimise the parameters for in vivo RNA probing in this unusual organism. We used two different probing agents with different nucleotide reactivity: NAI (2-methylnicotinic acid imidazolide) and DMS 
(dimethyl sulfate). NAI reacts with all four unpaired nucleotides while DMS selectively reacts with unpaired adenosine and cytosine. Neither of these chemicals has been used in $P$. falciparum before. In order to check if the chemicals could penetrate inside the parasite inside the host erythrocyte, we exposed an asynchronous culture of $P$. falciparum to NAI or DMS. After RNA extraction we performed a primer extension with a Cy5-conjugated primer targeting the 5.8S rRNA transcript. When a nucleotide has been modified by one of the chemicals, meaning that the nucleotide is unpaired, the reverse transcriptase will stall one nucleotide beforehand, stopping the elongation reaction (Fig. 1A). This can be seen on a denaturing polyacrylamide sequencing gel (Fig. 1B). Reading the sequence confirmed that it was identical to the PF3D7_0531800 gene sequence available on PlasmoDB (53). In NAI and DMS probed parasites, base reactivity was seen with all four nucleotides after NAI-probing, and with only adenosine and cytosine after DMSprobing. This indicated that the chemicals could penetrate into $P$. falciparum, and our probing strategy could investigate the RNA structures in $P$. falciparum.

To prepare the structurome, we then extracted the total RNA from two independent asynchronous parasite cultures treated with NAI, DMS, or DMSO only, as in figure 1, and used poly $(T)$ magnetic beads to enrich poly $(A)$ RNA. The enriched poly $(A)$ fraction represented $2-3.4 \%$ of the total RNA, which is commensurate with the literature for eukaryotic cells (54). The enriched fractions were then used to generate NGS libraries (Fig. 2A). The sequencing outputs were processed using StructureFold2 and RNAStructure for experimental-reactivity-constrained folding (55) to determine the secondary structure of the RNA molecules. Each replicate for each chemical treatment was analysed separately. After preliminary analysis, because the distribution of structure differences between replicates was marginal and the correlation between replicates and pooled samples was good (Supp Fig. 1), reads from both replicates were merged for subsequent analysis. To ensure structure calls of a high quality, mappings were filtered such that there were no more than 3 mismatches/indels from the reference and the first 5' base was matching. Coverage filtering also required transcripts with complete coverage between samples. Structural information was more comprehensive (and agreement between replicates was also better), for the NAI-probed datasets because all four unpaired nucleotides were detected. Subsequent analysis was therefore focused on the NAI dataset as the primary in vivo structurome.

\section{Structure of the NAl-probed dataset}

After filtering for low-coverage genes, the NAI structurome covered $51.23 \%$ of the total transcriptome of $P$. falciparum (2699 transcripts detected out of 5268 total). We first looked at the representation of the various families of RNA molecules. Within our dataset $95.5 \%$ of the transcripts were protein coding transcripts, $1.27 \%$ corresponded to mitochondrial transcripts (1.16\% mitochondrial rRNA, $0.11 \%$ mitochondrial $\mathrm{mRNA}$ ) and $0.94 \%$ were uncharacterised non-protein coding transcripts. Other types of non-coding RNA were also detected, $1.31 \%$ of snoRNA, $0.19 \%$ of snRNA and $0.34 \%$ of tRNA (Fig. 2B). Within the protein coding transcripts, nine were encoded in the apicoplast, a subcellular organelle that is unique to apicomplexans and represents a relic chloroplast (Supp Table 1).

As well as the different families of transcripts, we detected 114 transcripts with splice variants (Supp Table 2). However, since we used a short-read technology to 
sequence the library, it was impossible to associate all the reads within a variant transcript to one variant. Nevertheless, this overall analysis indicates that our in vivo chemical probing, RNA extraction and polyA selection were robust because all the different RNA families in $P$. falciparum were detected, with a strong bias towards mRNAs. If a long-read sequencing technique had been used, even the detection of transcript variants would likely have been possible.

Some RNA families have their function dictated by their structure. This is especially the case of tRNA. Within our dataset 9 tRNAs were identified. Using RNAStructure we were able to call their structures and 5 were similar to canonical eukaryotic tRNA molecules. Those tRNAs were for glutamic acid (PF3D7_0411600, PF3D7_0527700), methionine (PF3D7_1339100), asparagine (PF3D7_0714700), alanine (PF3D7_0620800) and proline (PF3D7_1339200) (Fig. 2C). The 3 other tRNAs, for valine (PF3D7_0312600), serine (PF̈3D7_0410100) and leucine (PF3D7_0620900), had structures that could not be associated with the canonical eukaryotic structures (Supp Fig. 2). Interestingly, the canonical tRNAs had slightly but significantly higher GC contents than the non-canonical ones (respective medians $56.34 \%$ and $54.55 \%$, Wilcoxon $p$-value $=0.02$ ). It is important to note, however, that tRNA structure is also dependent on nucleotide base modification such as $m^{2} A, m^{2} G$, pseudouridine, etc. (56). Those modifications have not been assessed in this study, so it is possible that they would influence these apparentlynoncanonical tRNA structures.

\section{Divergence between in vivo RNA structures and structures modelled in silico}

Using the RNAStructure algorithm, RNA structures can be predicted from primary sequence alone, or they can be predicted with the addition of base-pairing information gained experimentally.

Firstly, we sought to discover how much these two types of structures would differ in $P$. falciparum, i.e. how much new information was gained by performing the in vivo structurome? We calculated the ratio of bases that differed (i.e. that were paired versus unpaired) between each in-silico-predicted and probing-informed structure, and termed this ratio the 'divergence'. Most of the non-protein coding transcripts had relatively low divergence, meaning that they were minimally informed by probing (Table 2). This was expected for highly conserved, stereotypical structures like rRNAs and tRNAs - although the divergence for mitochondrial rRNAs, which are encoded in a highly fragmentary fashion in the $P$. falciparum mitochondrial genome, was notably greater than for nuclear-encoded rRNAs. By contrast, the divergence of protein coding transcripts was much higher: a median of 0.7067 for the nuclear-encoded mRNAs and 0.8117 for mitochondrial mRNAs (Table 2).

Since structure and function in non-protein coding transcripts, like tRNAs, are deeply intertwined, we then sought any relationship between folding complexity and biological function. Using the 'divergence' as a metric, we split the protein coding transcripts into 3 tiers: minimal value to $25^{\text {th }}$ percentile, $25^{\text {th }}$ to $75^{\text {th }}$ percentile and $75^{\text {th }}$ percentile to the highest value (Table 2), The list of transcripts was then submitted for gene ontology enrichment and using Revigo (57) we obtained broad families of GO terms and networks of terms belonging to a similar biological pathway. In tier 1, with the lowest 'divergence', we observed an enrichment of 25 terms, with the strongest enrichment in terms related to ribosomal functions. This was expected, since ribosomal RNA structures are highly conserved and are likely to be predicted accurately in silico with little alteration in $P$. falciparum. Other terms 
such as "macromolecular complex" were strongly enriched in tier 1, but they were not part of any network (Fig. 3A, Supp Table 3-1). Twenty-one terms were found in tier 2. Two terms were strongly enriched: 'translation' and 'cellular amide metabolism'; however, these stand alone. Two networks could be created: a large one around 'protein' and 'protein regulation' (composed only of lowly enriched words) and a second network restricted to two terms around RNA splicing (Fig. 3B, Supp Table 32). As for tier 3 , with the lowest level of identity between in vivo structures and in silico predictions, only 11 terms were enriched and 3 networks were made, the largest composed of 7 terms strongly enriched around different kinds of metabolism and the other networks around transport and complex molecule biosynthesis (Fig. 3C, Supp Table 3-3).

\begin{tabular}{|c|c|c|c|c|c|c|c|c|}
\hline & $\begin{array}{c}\text { Protein } \\
\text { coding }\end{array}$ & $\begin{array}{c}\text { mito m } \\
\text { RNA }\end{array}$ & $\begin{array}{c}\text { Uncharacterized } \\
\text { non protein coding }\end{array}$ & snoRNA & tRNA & rRNA & snRNA & $\begin{array}{c}\text { mito } \\
\text { rRNA }\end{array}$ \\
\hline \# transcript & 2549 & 3 & 25 & 35 & 9 & 8 & 5 & 36 \\
\hline Median & 0.7067 & 0.8117 & 0.5260 & 0.4414 & 0.1667 & 0.2204 & 0.4630 & 0.5439 \\
\hline Minimum & 0.04408 & 0.7480 & 0.07407 & 0.07500 & 0.0556 & 0.0202 & 0.1880 & 0.000 \\
\hline $\begin{array}{c}25 \% \\
\text { Percentile }\end{array}$ & 0.5995 & 0.7480 & 0.3428 & 0.2639 & 0.0972 & 0.0616 & 0.1916 & 0.3471 \\
\hline $\begin{array}{c}75 \% \\
\text { Percentile }\end{array}$ & 0.7874 & 0.8880 & 0.6914 & 0.6341 & 0.6001 & 0.3529 & 0.5636 & 0.7446 \\
\hline Maximum & 1.033 & 0.8880 & 0.9083 & 0.9176 & 0.7317 & 0.4706 & 0.6566 & 1.050 \\
\hline
\end{tabular}

Table 2: Distribution of the 'divergence' normalised by transcript length between base pairing determined by Structure-seq and in silico, categorised by family of RNA.

Secondly, we assessed the value of performing Structure-seq instead of just in silico prediction for modelling the shapes of RNA molecules accurately. We used ViennaFold on the $P$. falciparum transcriptome to fold the structures with or without reactivity data. We first aligned the dot/bracket sequences obtained from in silico folding with the Structure-seq-determined sequence of the U2 snRNA (PF3D7_1137000). It showed a difference in the pairing of 74 nucleotides out of 198 , meaning a $37.4 \%$ discrepancy in the structure determined in silico compared to in vivo (Fig. 4A). Structurally, the difference between the two transcripts is significant and only 3 loops are common to both structures (Fig. 4B). More interestingly, an RNA-appropriate measurement of Minimum Free Energy, MFEden (58), revealed that the Structure-seq U2 snRNA structure had a lower MFEden (-24.76 versus 15.50). Extending this to the whole dataset, we observed a similar effect in each RNA category (Table 3). Thus, structures called from Structure-seq are more likely to form and are more stable than the ones predicted only using an in silico prediction.

\begin{tabular}{|c|c|c|c|c|c|c|c|c|}
\hline & $\begin{array}{c}\text { protein } \\
\text { coding }\end{array}$ & $\begin{array}{c}\text { mito } \\
\text { mRNA }\end{array}$ & $\begin{array}{c}\text { uncharacterised } \\
\text { non protein } \\
\text { coding }\end{array}$ & snoRNA & tRNA & rRNA & snRNA & $\begin{array}{c}\text { mito } \\
\text { rRNA }\end{array}$ \\
\hline $\begin{array}{c}\text { Structure- } \\
\text { seq }\end{array}$ & -20.5 & -27.0 & -29.8 & -29.6 & -63 & -31.1 & -37.9 & -25 \\
\hline in silico & 14.1 & 10.3 & 10.7 & 10.2 & -8.8 & 3.2 & 10.7 & 2.7 \\
\hline
\end{tabular}

Table 3: MFEden average for every type of RNA molecule. All MFEden values per transcript are in Supp Table 4. 


\section{Effect of $\mathrm{A} / \mathrm{T}$ bias in the structurome of $\boldsymbol{P}$. falciparum by comparison with an A/T-balanced Plasmodium species}

The Plasmodium genus offers the possibility to compare very different genome compositions. $P$. falciparum is $>80 \% \mathrm{~A} / \mathrm{T}$ rich while $P$. knowlesi, a macaque Plasmodium species which also infects humans, is $62.5 \% \mathrm{~A} / \mathrm{T}$ rich (59). In order to assess whether genome base composition could affect the structurome, we compared the in silico folding of $P$. falciparum and $P$. knowlesi structuromes. Firstly, we assessed the stability of the two predicted structuromes by comparing the average free energy (MFEden (58)) of all folded structures: $P$. falciparum had a significantly higher MFEden than $P$. knowlesi (respectively 14.03 and 7.1, t-test $p$ value $<0.0001)$. Secondly, we looked at the occurrence of various structures by comparing the length-normalised number of nucleotides participating in a structure (e.g. a hairpin or bulge) in each $P$. falciparum transcript with their $P$. knowlesi orthologs. The structurome of $P$. falciparum had significantly more nucleotides involved in hairpins and multi-stem loops than the structurome of $P$. knowlesi (t-test, $p$-value $<0.0001$ ), whereas $P$. knowlesi had significantly more nucleotides involved in stems and bulges (t-test, $p$-value <0.0001) (Supp Table 5).

\section{RNA structures affect translation efficiency in a stage specific manner}

Figure 2 shows that various families of RNAs display very conserved structures that are related to their particular functions. This is clearly true for structural RNAs (tRNAs, rRNAs, etc.), but it may also be true for mRNAs, whose main function is to be translated into proteins. The stability of the transcript and the efficiency of its translation could both be affected by mRNA structure. Therefore, if mRNA structures can vary with varying cellular conditions, transcript and protein abundance may both vary accordingly.

We first explored the concept that transcript abundance could vary with cellular conditions due to changes in mRNA structure. For this, we used published microarray and transcriptomic datasets from $P$. falciparum under physiological stresses like hyperoxia (49), or drug exposure like chloroquine treatment (50). These datasets reveal which transcripts are upregulated and downregulated in each condition. We compared the most differentially expressed genes in each condition with the structurome dataset, to see if they were particularly structured or unstructured. Neither the overall degree of 'divergence' nor the representation of diverse structures (the proportion of hairpins, loops, etc), correlated with differential expression under stress (data not shown).

Secondly, we looked at the effect of RNA structures on translation efficiency (TE). To establish the translation efficiency for each transcript in our dataset, we extracted from previously published work (51) the average level of ribosome attachment per transcript, determined by Ribo-seq. We obtained TE for the transcriptome of rings, early and late trophozoites and schizonts. Applying a linear regression model, we compared TE with transcript structuredness (the 'NAI ratio', i.e. the ratio of paired to unpaired bases) and also the proportion of different motifs such as stems, hairpins, multi-loop stems and bulges (Fig. 5). We observed no correlation between the transcript-length-normalised 'structuredness' and TE in any of the four stages (Fig. 5A). Therefore, there was no overall relationship between the proportion of paired bases in a transcript and the efficiency of its translation. However, breaking 
down the different possible structures (Fig. 5B-E) we observed a positive correlation of TE with the presence of stems and a negative correlation between TE and hairpins, multi-loop stems and bulges. The correlations were only seen in the more mature parasite stages, late trophozoites and schizonts.

\section{RNA decay is mediated by the same structures across the cell cycle}

The final critical part of an RNA molecule's life inside the cell is its rate of decay. Following a similar reasoning as for translation efficiency, we extracted RNA decay values from data published by Painter et al. (52) and tested for any correlation between RNA decay and RNA secondary structures in the different cell cycle stages (rings, early and late trophozoites and schizonts). For this, we applied a linear regression model to compare RNA decay (the higher the value, the less stable the molecule is) to the number of different structures per transcript. We observed no correlation between RNA decay and RNA secondary structures involving high amounts of base pairing: stems and hairpins (Fig. 6A, B). However, when nucleotides were more exposed, as in bulges and multi-stem loops (Fig. 6C, D) we observed correlations between RNA decay and the presence of those structures in all erythrocytic stages (except for multi-stem loops in ring-stage) (Fig. 6C).

\section{Discussion}

In this study, we produced the very first SHAPE-seq-determined structurome of a parasitic protozoan, $P$. falciparum. Firstly, we demonstrated that it is possible to use probing chemicals on the parasite within the host erythrocyte, meaning that the chemicals can get through the host cell membrane, the parasitophorous vacuole and the parasite membrane. The fact that we could also detect mitochondrial and apicoplast transcripts shows that the chemicals also passed through the inner organellar membranes. Thus, we obtained in vivo structures for about half the transcripts in the transcriptome, including structural and noncoding RNAs as well as mRNAs.

Most of the structural RNAs had stereotypical structures, although a subset of tRNAs did not model stereotypically - an interesting observation that merits further investigation. Amongst mRNAs, the in vivo structures tended to have lower free energy, making them more likely to form, than the equivalent structures predicted in silico, thus demonstrating the value of the experimental approach. Highly conserved RNAs such as ribosomal RNAs tended to be predicted well, whereas classes of genes whose structures were poorly predicted in silico included genes involved in metabolism, biosynthesis of complex molecules, and transport. These genes may have become particularly divergent in $P$. falciparum due to its parasitic intracellular lifestyle.

We also took advantage of the striking disparities in genome composition in the Plasmodium genus to see if the extreme base composition of the $P$. falciparum transcriptome might have an impact on the different structures formed, by comparing the predicted structurome of $P$. knowlesi with that of $P$. falciparum. We observed that in $P$. falciparum transcripts were more prone to form structures like hairpins and multi-stem loops, whereas a more balanced genome like $P$. knowlesi gave rise to orthologous transcripts with more stems. Overall, $P$. falciparum transcripts were predicted to fold with higher free energy than their orthologs in $P$. knowlesi, suggesting that an $\mathrm{A} / \mathrm{U}$-biased transcriptome is less stable. However, this might be 
mitigated by in vivo factors, since we also found that overall the $P$. falciparum in vivo structurome had lower free energy than was predicted in silico. It would be interesting in future to perform an in vivo structurome of $P$. knowlesi and see whether in vivo transcripts tend towards greater stability in this species as well.

To examine how the RNA structurome might influence parasite biology, we looked at the impacts of the different structures on translation efficiency and RNA decay. We showed that RNA structures had no impact on translation efficiency in rings and early trophozoites, two stages of the erythrocytic cycle that are translationally less active $(51,60)$. On the contrary, late trophozoites and schizonts are more translationally active and negative correlations between translation efficiency and complex structures like hairpins, multi-loop stem and bulges was observed. Recently, we studied a particularly stable RNA structure in $P$. falciparum, the RNA G-quadruplex, and demonstrated that it likewise had a negative impact on translation (61). We also observed a positive correlation between translation efficiency and a simpler RNA structure, stems. This is consistent with the idea that stem structures can facilitate ribosome attachment, whereas other complex structures can inhibit it (62). When we associate these results with the negative correlation between structures and RNA decay, we can hypothesise that RNA structures influence protein expression on at least two levels: the rate of translation and the degree of transcript stability. Similar effects were previously demonstrated in human cell lines, and furthermore, RNA base modifications were also found to be important, impacting protein expression by changing the functional half-life of transcripts (63). This area has been little explored in $P$. falciparum as yet, but a recent study of base modification in $P$. falciparum likewise implicated RNA base modification in protein expression (64).

This study focuses on RNA structure in the erythrocytic life cycle of Plasmodium parasites. Two more parts of the full life cycle remain to be studied in future: the liver stage and the sexual cycle which happens in the host vector, a mosquito. The liver stages are particularly interesting because two human-infective Plasmodium species, $P$. vivax and $P$. ovale, can make hypnozoites - a dormant form that can stay inside the liver of infected people for months and then reactivate to cause disease (65). Currently the biology of hypnozoites remains complicated to investigate and to comprehend. However, translational repression may be key to long-term dormancy, so the RNA structurome of hypnozoites could be intriguing. A more accessible stage, the gametocyte, also shows dormancy (albeit a dormancy that lasts days rather than months), and female gametocytes are known to translationally repress hundreds of transcripts (66). Once in the mosquito midgut, the translational repression is lifted, and we can hypothesise that the change of temperature between a warm-blooded host and a non-thermoregulatory insect would impact RNA structures, playing a role in lifting the repression.

Within the mosquito, the sexual life cycle encompasses four different life stages for which no RNA structures are known. This step of the life cycle is crucially important in completion of the transmission chain and also in generating genetic diversity (67). Recent progress in single cell RNA sequencing give us the opportunity to look at the transcriptome of single parasites at all life cycle stages, including those in the host vector (68). In parallel, novel RNA structure determination techniques, like SHAPE-MaP-seq associated with the latest algorithms like DRACO, would make the determination of single cell RNA structures possible (69). Other technological breakthroughs like SMRT-seq (Single Molecule, Real Time) associated with SHAPEMaP would allow access to transcriptional variant structures (70), which were not 
possible to analyse in this study, and this could shed new light on post-transcriptional regulation in Plasmodium. In conclusion, this work paves the way to decipher another level of complexity in the molecular biology of Plasmodium parasites.

\section{Data availability}

Raw data (FASTQ) from sequencing for each sample is available from the European Nucleotide Archive (ENA) under accession PRJEB44384.

\section{Funding Information}

UK Medical Research Council (grants MR/K000535/1 and MR/L008823/1) to CJM, Royal Society Kan Tong Po fellowship, Shenzhen Basic Research Project (JCYJ20180507181642811), Research Grants Council of the Hong Kong SAR, China Projects CityU 11101519, CityU 11100218, N_CityU110/17, CityU 21302317, Croucher Foundation Project No. 9509003, 9500030, State Key Laboratory of Marine Pollution Director Discretionary Fund, City University of Hong Kong projects $6000711,7005503,9680261,9667222$ to CKK. The funders had no role in study design, data collection and interpretation, or the decision to submit the work for publication.

\section{Author Contributions}

FD performed experiments and data analysis, made the figures and wrote the manuscript, AE performed the bioinformatic Structure-seq analysis, JZ performed the structure probing gel assay and Structure-seq library preparation and sequencing, and participated in the writing, CKK and CJM conceptualised the paper and wrote the manuscript.

\section{Acknowledgements}

We would like to thank Dr. Betty Chung from the Cambridge Pathology Department for her help with the transcription efficiency data processing.

\section{Bibliography}

1. Mathews,D.H., Sabina,J., Zuker,M. and Turner,D.H. (1999) Expanded sequence dependence of thermodynamic parameters improves prediction of RNA secondary structure. J. Mol. Biol., 288, 911-40.

2. Sharp,P.A. (2009) The Centrality of RNA. Cell, 136, 577-580.

3. Cech,T.R. and Steitz,J.A. (2014) The noncoding RNA revolution-trashing old rules to forge new ones. Cell, 157, 77-94.

4. Wan,Y., Kertesz,M., Spitale,R.C., Segal,E. and Chang,H.Y. (2011) Understanding the transcriptome through RNA structure. Nat. Rev. Genet., 12, 641-655.

5. Vandivier,L.E., Anderson,S.J., Foley,S.W. and Gregory,B.D. (2016) The Conservation and Function of RNA Secondary Structure in Plants. Annu. Rev. Plant Biol., 67, 463-488.

6. Sun,L., Fazal,F.M., Li,P., Broughton,J.P., Lee,B., Tang,L., Huang,W., Kool,E.T., Chang,H.Y. and Zhang,Q.C. (2019) RNA structure maps across mammalian cellular compartments. Nat. Struct. Mol. Biol., 26, 322-330.

7. Casas-Vila,N., Sayols,S., Pérez-Martínez,L., Scheibe,M. and Butter,F. (2020) The RNA fold interactome of evolutionary conserved RNA structures in S. cerevisiae. Nat. Commun., 11, 1-12.

8. Hasler,D. and Meister,G. (2016) From tRNA to miRNA: RNA-folding contributes to correct entry into noncoding RNA pathways. FEBS Lett., 590, 2354-63. 
9. Wachter,A. (2010) Riboswitch-mediated control of gene expression in eukaryotes. RNA Biol., 7.

10. Wilkinson,K.A., Merino,E.J. and Weeks,K.M. (2006) Selective 2'-hydroxyl acylation analyzed by primer extension (SHAPE): quantitative RNA structure analysis at single nucleotide resolution. Nat. Protoc., 1, 1610-6.

11. Spitale,R.C., Crisalli,P., Flynn,R.A., Torre,E.A., Kool,E.T. and Chang,H.Y. (2013) RNA SHAPE analysis in living cells. Nat. Chem. Biol., 9, 18-20.

12. Westhof,E. and Romby,P. (2010) The RNA structurome: high-throughput probing. Nat. Methods, 7, 965-967.

13. Ding,Y., Tang,Y., Kwok,C.K., Zhang,Y., Bevilacqua,P.C. and Assmann,S.M. (2014) In vivo genome-wide profiling of RNA secondary structure reveals novel regulatory features. Nature, 505, 696-700.

14. Kwok,C.K., Tang,Y., Assmann,S.M. and Bevilacqua,P.C. (2015) The RNA structurome: transcriptome-wide structure probing with next-generation sequencing. Trends Biochem. Sci., 40, 221-32.

15. Zhao,J., Qian,X., Yeung,P.Y., Zhang,Q.C. and Kwok,C.K. (2019) Mapping In Vivo RNA Structures and Interactions. Trends Biochem. Sci., 44, 555-556.

16. Watts,J.M., Dang,K.K., Gorelick,R.J., Leonard,C.W., Bess,J.W., Swanstrom,R., Burch,C.L. and Weeks,K.M. (2009) Architecture and secondary structure of an entire HIV-1 RNA genome. Nature, 460, 711-6.

17. Li,P., Wei,Y., Mei,M., Tang,L., Sun,L., Huang,W., Zhou,J., Zou,C., Zhang,S., Qin,C.F., et al. (2018) Integrative Analysis of Zika Virus Genome RNA Structure Reveals Critical Determinants of Viral Infectivity. Cell Host Microbe, 24, 875886.e5.

18. Huber,R.G., Lim,X.N., Ng,W.C., Sim,A.Y.L., Poh,H.X., Shen,Y., Lim,S.Y., Sundstrom,K.B., Sun,X., Aw,J.G., et al. (2019) Structure mapping of dengue and Zika viruses reveals functional long-range interactions. Nat. Commun., 10.

19. Simon,L.M., Morandi,E., Luganini,A., Gribaudo,G., Martinez-Sobrido,L., Turner,D.H., Oliviero,S. and Incarnato,D. (2019) In vivo analysis of influenza A mRNA secondary structures identifies critical regulatory motifs. Nucleic Acids Res., 47, 7003-7017.

20. Manfredonia,I., Nithin,C., Ponce-Salvatierra,A., Ghosh,P., Wirecki,T.K., Marinus,T., Ogando,N.S., Snijder,E.J., van Hemert,M.J., Bujnicki,J.M., et al. (2020) Genome-wide mapping of SARS-CoV-2 RNA structures identifies therapeutically-relevant elements. Nucleic Acids Res., 10.1093/nar/gkaa1053.

21. Sun,L., Li,P., Ju,X., Rao,J., Huang,W., Ren,L., Zhang,S., Xiong,T., Xu,K., Zhou,X., et al. (2021) In vivo structural characterization of the SARS-CoV-2 RNA genome identifies host proteins vulnerable to repurposed drugs. Cell, 184, 1865-1883.e20.

22. Ziv,O., Price,J., Shalamova,L., Kamenova,T., Goodfellow,I., Weber,F. and Miska,E.A. (2020) The Short- and Long-Range RNA-RNA Interactome of SARSCoV-2. Mol. Cell, 80, 1067-1077.e5.

23. Huston,N.C., Wan,H., Strine,M.S., de Cesaris Araujo Tavares,R., Wilen,C.B. and Pyle,A.M. (2021) Comprehensive in vivo secondary structure of the SARS-CoV2 genome reveals novel regulatory motifs and mechanisms. Mol. Cell, 81, 584598.e5.

24. Ling Yang,S., DeFalco,L., Anderson,D.E., Zhang,Y., Aw,A.J., Lim,Y., Xin Ni,L., Yee Tan,K., Zhang,T., Chawla,T., et al. (2021) Comprehensive mapping of SARS-CoV-2 interactions in vivo reveals 1 functional virus-host interactions 23. bioRxiv, 10.1101/2021.01.17.427000. 
25. Deng,H., Cheema,J., Zhang,H., Woolfenden,H., Norris,M., Liu,Z., Liu,Q., Yang,X., Yang,M., Deng,X., et al. (2018) Rice In Vivo RNA Structurome Reveals RNA Secondary Structure Conservation and Divergence in Plants. Mol. Plant, 11, 607-622.

26. Rouskin,S., Zubradt,M., Washietl,S., Kellis,M. and Weissman,J.S. (2014) Genome-wide probing of RNA structure reveals active unfolding of mRNA structures in vivo. Nature, 505, 701-705.

27. Guo,J.U. and Bartel,D.P. (2016) RNA G-quadruplexes are globally unfolded in eukaryotic cells and depleted in bacteria. Science (80-. )., 353.

28. Incarnato,D., Neri,F., Anselmi,F. and Oliviero,S. (2014) Genome-wide profiling of mouse RNA secondary structures reveals key features of the mammalian transcriptome. Genome Biol., 15, 491.

29. Wu,X. and Bartel,D.P. (2017) Widespread Influence of 3'-End Structures on Mammalian mRNA Processing and Stability. Cell, 169, 905-917.e11.

30. WHO (2018) World malaria report 2018.

31. Su,Z., Tang,Y., Ritchey,L.E., Tack,D.C., Zhu,M., Bevilacqua,P.C. and Assmann,S.M. (2018) Genome-wide RNA structurome reprogramming by acute heat shock globally regulates mRNA abundance. Proc. Natl. Acad. Sci. U. S. A., 115, 12170-12175.

32. Righetti,F., Nuss,A.M., Twittenhoff,C., Beele,S., Urban,K., Will,S., Bernhart,S.H., Stadler,P.F., Dersch,P. and Narberhaus,F. (2016) Temperature-responsive in vitro RNA structurome of Yersinia pseudotuberculosis. Proc. Natl. Acad. Sci. U. S. A., 113, 7237-7242.

33. Twittenhoff,C., Brandenburg,V.B., Righetti,F., Nuss,A.M., Mosig,A., Dersch,P. and Narberhaus,F. (2020) Lead-seq: Transcriptome-wide structure probing in vivo using lead(II) ions. Nucleic Acids Res., 48, E71-E71.

34. Waters,A.P., Syin,C. and McCutchan,T.F. (1989) Developmental regulation of stage-specific ribosome populations in Plasmodium. Nature, 342, 438-40.

35. Gunderson,J.H., Sogin,M.L., Wollett,G., Hollingdale,M., de la Cruz,V.F., Waters,A.P. and McCutchan,T.F. (1987) Structurally distinct, stage-specific ribosomes occur in Plasmodium. Science, 238, 933-7.

36. Fang,J. and McCutchan, T.F. (2002) Thermoregulation in a parasite's life cycle. Nature, 418, 742.

37. Fang,J., Zhou,H., Rathore,D., Sullivan,M., Su,X.Z. and McCutchan,T.F. (2004) Ambient glucose concentration and gene expression in Plasmodium falciparum. Mol. Biochem. Parasitol., 133, 125-129.

38. John Rogers,M., Gutell,R.R., Damberger,S.H., Li,J., Mcconkey,G.A., Waters,A.P. and McCutchan,T.F. (1996) Structural features of the large subunit rRNA expressed in Plasmodium falciparum sporozoites that distinguish it from the asexually expressed large subunit rRNA. RNA, 2, 134-145.

39. Velichutina,I. V., Rogers,M.J., McCutchan,T.F. and Liebman,S.W. (1998) Chimeric rRNAs containing the GTPase centers of the developmentally regulated ribosomal $r$ RNAs of Plasmodium falciparum are functionally distinct. $R N A, 4,594-602$.

40. Gardner,M.J., Hall,N., Fung,E., White,O., Berriman,M., Hyman,R.W., Carlton,J.M., Pain,A., Nelson,K.E., Bowman,S., et al. (2002) Genome sequence of the human malaria parasite Plasmodium falciparum. Nature, 419, 498-511.

41. Trager,W. and Jensen,J.B. (1976) Human malaria parasites in continuous culture. Science, 193, 673-5.

42. Kwok,C.K., Ding,Y., Tang,Y., Assmann,S.M. and Bevilacqua,P.C. (2013) 
Determination of in vivo RNA structure in low-abundance transcripts. Nat. Commun., 4, 2971.

43. Yeung,P.Y., Zhao,J., Chow,E.Y.C., Mou,X., Hong,H.Q., Chen,L., Chan,T.F. and Kwok,C.K. (2019) Systematic evaluation and optimization of the experimental steps in RNA G-quadruplex structure sequencing. Sci. Rep., 9, 1-12.

44. Davis,M.P.A., van Dongen,S., Abreu-Goodger,C., Bartonicek,N. and Enright,A.J. (2013) Kraken: A set of tools for quality control and analysis of high-throughput sequence data. Methods, 63, 41-49.

45. Langmead,B., Wilks,C., Antonescu,V. and Charles,R. (2019) Scaling read aligners to hundreds of threads on general-purpose processors. Bioinformatics, 35, 421-432.

46. Tack,D.C., Tang,Y., Ritchey,L.E., Assmann,S.M. and Bevilacqua,P.C. (2018) StructureFold2: Bringing chemical probing data into the computational fold of RNA structural analysis. Methods, 143, 12-15.

47. Reuter,J.S. and Mathews,D.H. (2010) RNAstructure: software for RNA secondary structure prediction and analysis. BMC Bioinformatics, 11, 129.

48. Lorenz,R., Bernhart,S.H., Höner Zu Siederdissen,C., Tafer,H., Flamm,C., Stadler,P.F. and Hofacker,I.L. (2011) ViennaRNA Package 2.0. Algorithms Mol. Biol., 6, 26.

49. Torrentino-Madamet,M., Alméras,L., Desplans,J., Priol,Y. Le, Belghazi,M., Pophillat,M., Fourquet,P., Jammes,Y. and Parzy,D. (2011) Global response of Plasmodium falciparum to hyperoxia: A combined transcriptomic and proteomic approach. Malar. J., 10, 1-15.

50. Untaroiu,A.M., Carey,M.A., Guler,J.L. and Papin,J.A. (2019) Leveraging the effects of chloroquine on resistant malaria parasites for combination therapies. BMC Bioinformatics, 20, 1-9.

51. Caro,F., Ahyong,V., Betegon,M. and DeRisi,J.L. (2014) Genome-wide regulatory dynamics of translation in the Plasmodium falciparum asexual blood stages. Elife, 3, 1-24.

52. Painter,H.J., Chung,N.C., Sebastian,A., Albert,I., Storey,J.D. and Llinás,M. (2018) Genome-wide real-time in vivo transcriptional dynamics during

53. Plasmodium falciparum blood-stage development. Nat. Commun., 9, 2656.Aurrecoechea,C., Brestelli,J., Brunk,B.P., Dommer,J., Fischer,S., Gajria,B., Gao,X., Gingle,A., Grant,G., Harb,O.S., et al. (2009) PlasmoDB: a functional genomic database for malaria parasites. Nucleic Acids Res., 37, D539-D543.

54. Westermann,A.J., Gorski,S.A. and Vogel,J. (2012) Dual RNA-seq of pathogen and host. Nat. Rev. Microbiol., 10, 618-630.

55. Ritchey,L.E., Su,Z., Tang,Y., Tack,D.C., Assmann,S.M. and Bevilacqua,P.C. (2017) Structure-seq2: sensitive and accurate genome-wide profiling of RNA structure in vivo. Nucleic Acids Res., 45, e135.

56. Jackman,J.E. and Alfonzo,J.D. (2013) Transfer RNA modifications: Nature's combinatorial chemistry playground. Wiley Interdiscip. Rev. RNA, 4, 35-48.

57. Supek,F., Bošnjak,M., Škunca,N. and Šmuc,T. (2011) REVIGO Summarizes and Visualizes Long Lists of Gene Ontology Terms. PLoS One, 6, e21800.

58. Trotta,E. (2014) On the normalization of the minimum free energy of RNAs by sequence length. PLoS One, 9.

59. Pain,A., Böhme,U., Berry,A.E., Mungall,K., Finn,R.D., Jackson,A.P., Mourier,T., Mistry,J., Pasini,E.M., Aslett,M.A., et al. (2008) The genome of the simian and human malaria parasite Plasmodium knowlesi. Nature, 455, 799-803. 
60. Bunnik,E.M., Batugedara,G., Saraf,A., Prudhomme,J., Florens,L. and Le Roch,K.G. (2016) The mRNA-bound proteome of the human malaria parasite Plasmodium falciparum. Genome Biol., 17, 1-18.

61. Dumetz,F., Chow,E.Y., Harris,L.M., Umar,M.I., Jensen,A., Chung,B., Chan,T.F., Merrick,C.J. and Kwok,C.K. (2021) G-quadruplex RNA motifs influence gene expression in the malaria parasite Plasmodium falciparum. bioRxiv, 10.1101/2021.03.08.434398.

62. Kramer,M.C. and Gregory,B.D. (2018) Does RNA secondary structure drive translation or vice versa? Nat. Struct. Mol. Biol., 25, 641-643.

63. Mauger,D.M., Cabral,B.J., Presnyak,V., Su,S. V., Reid,D.W., Goodman,B., Link,K., Khatwani,N., Reynders,J., Moore,M.J., et al. (2019) mRNA structure regulates protein expression through changes in functional half-life. Proc. Natl. Acad. Sci. U. S. A., 116, 24075-24083.

64. Baumgarten,S., Bryant,J.M., Sinha,A., Reyser,T., Preiser,P.R., Dedon,P.C. and Scherf,A. (2019) Transcriptome-wide dynamics of extensive m6A mRNA methylation during Plasmodium falciparum blood-stage development. Nat. Microbiol., 4, 2246-2259.

65. White,N.J. and Imwong,M. (2012) Relapse. In Advances in Parasitology.pp. 113150.

66. Guerreiro,A., Deligianni,E., Santos,J.M., Silva,P.A.G.C., Louis,C., Pain,A., Janse,C.J., Franke-Fayard,B., Carret,C.K., Siden-Kiamos, I., et al. (2014) Genome-wide RIP-Chip analysis of translational repressor-bound mRNAs in the Plasmodium gametocyte. Genome Biol., 15, 1-16.

67. Arez,A.P., Pinto,J., Pålsson,K., Snounou,G., Jaenson,T.G.T. and do Rosário,V.E. (2003) Transmission of mixed Plasmodium species and Plasmodium falciparum genotypes. Am. J. Trop. Med. Hyg., 68, 161-8.

68. Bogale,H.N., Pascini,T. V., Kanatani,S., Sá,J.M., Wellems,T.E., Sinnis,P., VegaRodríguez,J. and Serre,D. (2021) Transcriptional heterogeneity and tightly regulated changes in gene expression during Plasmodium berghei sporozoite development. Proc. Natl. Acad. Sci., 118, e2023438118.

69. Morandi,E., Manfredonia,I., Simon,L.M., Anselmi,F., van Hemert,M.J., Oliviero,S. and Incarnato,D. (2021) Genome-scale deconvolution of RNA structure ensembles. Nat. Methods, 18, 249-252.

70. Mays,A.D., Schmidt,M., Graham,G., Tseng,E., Baybayan,P., Sebra,R., Sanda,M., Mazarati,J.B., Riegel,A. and Wellstein,A. (2019) Single-molecule real-time (SMRT) full-length RNA-sequencing reveals novel and distinct mRNA isoforms in human bone marrow cell subpopulations. Genes (Basel)., 10, 1-17. 


\section{Figure legends}

Figure 1: In vivo probing of RNA structures in $P$. falciparum

(A) Schematic of RNA structure probing. An asynchronous parasite culture was split into two, one exposed to NAI (+NAI) and the other exposed to DMSO as control ($\mathrm{NAI}$ ). Reverse transcription (RT) was performed using a 5'-Cy5 conjugated primer (dark red star) specific for the 5.8S rRNA of $P$. falciparum (in green). During RT, nucleotides that have been modified by NAI in the +NAI samples (red dots) will stall the reverse transcription, interrupting the elongation process (black line). Finally, elongation cDNA products are resolved on a PAGE gel. (B) Sequencing gel showing the 5.8S rRNA gene of $P$. falciparum. Lanes 1 to 4 are sequencing lanes where one of the four nucleotides in the reaction mix has been switched with the dideoxynucleotide, provoking RT-termination. Lanes 5 and 6 represent respectively DMS and NAI modifications on 5.8S rRNA.

Figure 2: RNA structurome of $\boldsymbol{P}$. falciparum using Structure-seq (A) Schematic showing the Structure-seq pipeline. An asynchronous parasite culture was split into two, one exposed to NAI (+NAI) and the other exposed to DMSO as control (-NAI). Polyadenylated-enriched RNA fractions were then used to prepare the sequencing libraries in parallel. Fragmentation was performed to generate an average length ( $250 \mathrm{nt}$ ) of RNA for sequencing followed by 3' dephosphorylation, which replaces the 2'3' cyclic phosphate group with a 3'-OH group for 3' adapter ligation. Then a 3' adapter (black line) was ligated to the 3'-OH group of dephosphorylated RNA. Next, RT of RNA molecules (orange line) was carried out using a designed reverse primer (dark blue line) at the 5' end. During RT, nucleotides that had been modified by NAI in the +NAI samples (red dots) would stall the reverse transcription, interrupting the elongation process (pink line). Later, a 5' adaptor (light blue line) was added by ssDNA ligation at the 3' end of the newly synthesised cDNA strand (pink line). Finally, forward primers (brown+light blue line) and reverse primers (dark blue+red+green line) with different indexes were added by PCR for NGS and bioinformatic analysis. Reads were aligned to the PF3D7 reference genome and subsequently analysed with StructureFold2 to determine the structural reactivity sequence (transcribed as a dot/bracket sequence denoting paired/unpaired nucleotides), and with RNAStructure to predict the folding. (B) Representation of the diversity of the dataset regarding RNA families. (C) Structureseq derived structures of glutamic acid (PF3D7_0411600), methionine (PF3D7_1339100), asparagine (PF3D7_0714700), alanine (PF3D7_0620800) and proline (PF3D7_1339200) tRNAs, all of which are similar to canonical tRNA structures.

Figure 3: GO terms enrichment networks for protein coding transcripts, tier 1 to 3.

All networks represent the GO enrichment provided by REVIGO for (A) tier 1, (B) tier 2 and $(\mathbf{C})$ tier 3 . The brighter the red, the more enriched the term is. Detailed values are available in supp table 3.

Figure 4: Difference in U2 snRNA structure determined by Structure-seq versus in silico prediction 
(A) U2 snRNA (PF3D7_1137000) reactivity sequence alignment, comparing in vitro probing using NAI and in silico prediction using the same constraint parameters. The first line shows the nucleotide sequence, the second line shows the in silico predicted dot/bracket sequence and the third line, the Structure-seq dot/bracket sequence extracted from reactivity measurement. A dot shows a nucleotide not paired with any other nucleotide. An open bracket shows a nucleotide paired with another and starting a structural motif, while a closed bracket shows a nucleotide paired with another but finishing a structural motif. Red-highlighted dot or bracket indicates a change of pairing between the NAI and in silico predicted structural sequence. A green-highlighted bracket indicates a change in motif orientation between the NAI and in silico predicted structural sequence. Boxes with roman numerals indicate the conserved structures between the two shapes. (B) U2 snRNA structure predicted in silico from RNA sequence alone, on the left, and with the addition of reactivity data on the right. The red and green colouring have the same meaning as in $(A)$.

Figure 5: Impact of various RNA motifs on transcription efficiency across the life cycle of $\boldsymbol{P}$. falciparum

The columns represent the different erythrocytic life stages of $P$. falciparum and the rows represent the correlation between transcription efficiency and $(\mathbf{A})$ the transcript structuredness normalised by the transcript length, (B) the number of stems, (C) the number of hairpins, (D) the number of multi-stem loops and $(\mathbf{E})$ the number of bulges. Statistically significant positive correlations have a $p$-value framed in green and statistically significant negative correlations have a $p$-value framed in red.

\section{Figure 6: Correlation between RNA secondary structures and RNA decay} across the life cycle of $P$. falciparum

The columns represent the different erythrocytic life stages of $P$. falciparum and the rows represent the correlation between RNA decay and $(\mathbf{A})$ the number of stems, $(\mathbf{B})$ the number of hairpins, (C) the number of multi-stem loops and (D) the number of bulges. Statistically significant negative correlations have a $p$-value framed in red.

Supplementary figure 1: DMS and NAI sample pooling data (A) Distribution of structure distance between samples (unpooled and pooled) and the DMSO control and (B) structural similarity correlation plot.

Supplementary figure 2: Structure-seq folding of three non-canonical shape tRNA: (A) valine tRNA (PF3D7_0312600), (B) serine tRNA (PF3D7_0410100) and (C) leucine (PF3D7_0620900)

Supplementary table 1: Complete and detailed list of transcripts found during the Structure-seq analysis. The file contains all the genomic information, product description, gene type, transcript length, GC and AT content, reactivity for each replicated of DMS and NAI experiment plus the combined one, and the gene ontology information.

Supplementary table 2: Table of transcript variants. Same information available as in Supplementary table 1. 
Supplementary table 3: REVIGO output for clustering of GO terms. Each tab represents the data per tier of divergence.

Supplementary table 4: Table of free energy and transcript structures from Structure-seq and in silico analysis.

Supplementary table 5: List of the $P$. falciparum transcripts and their $P$. knowlesi orthologs with in silico folding information normalised by transcript length. 

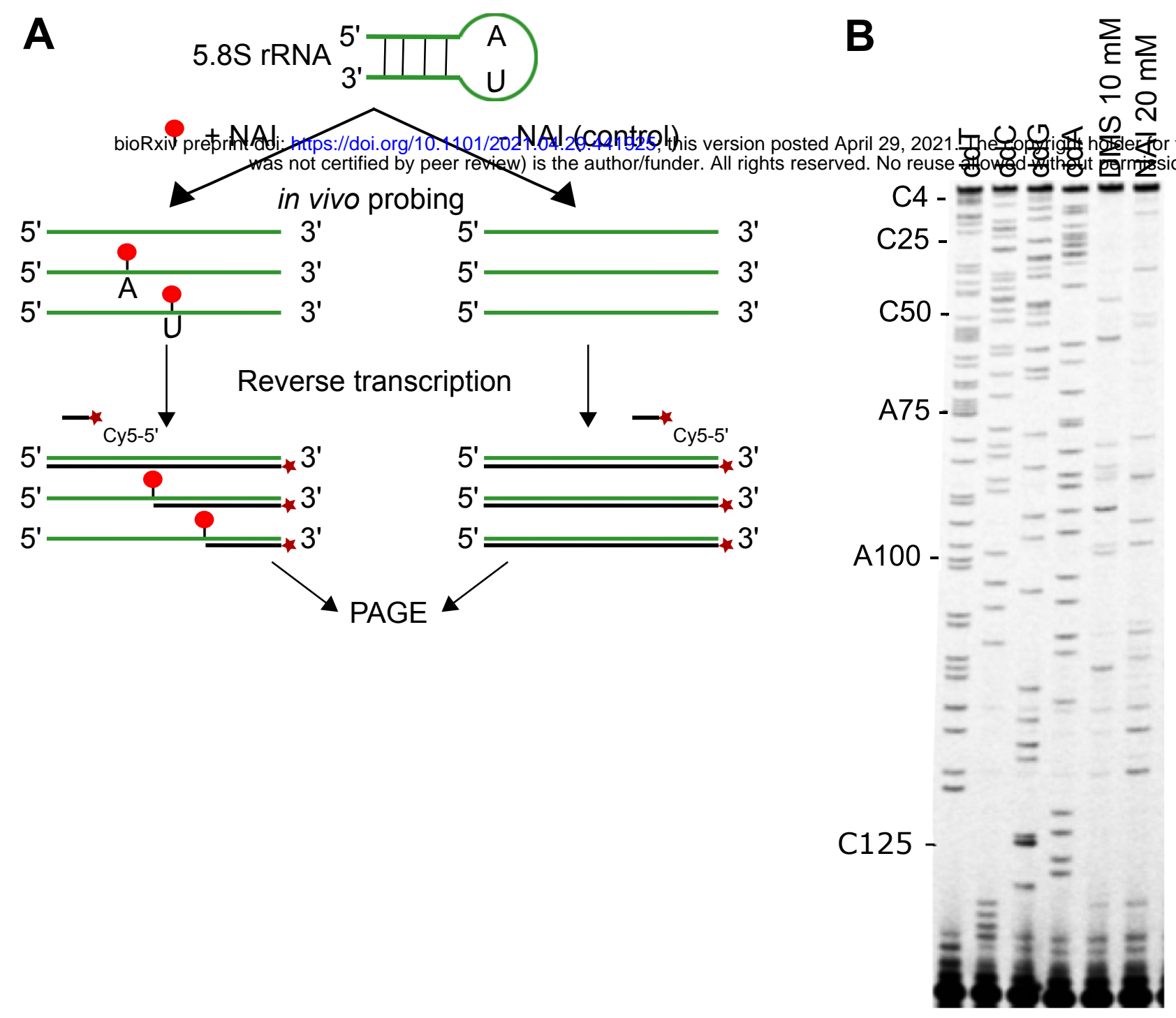
A

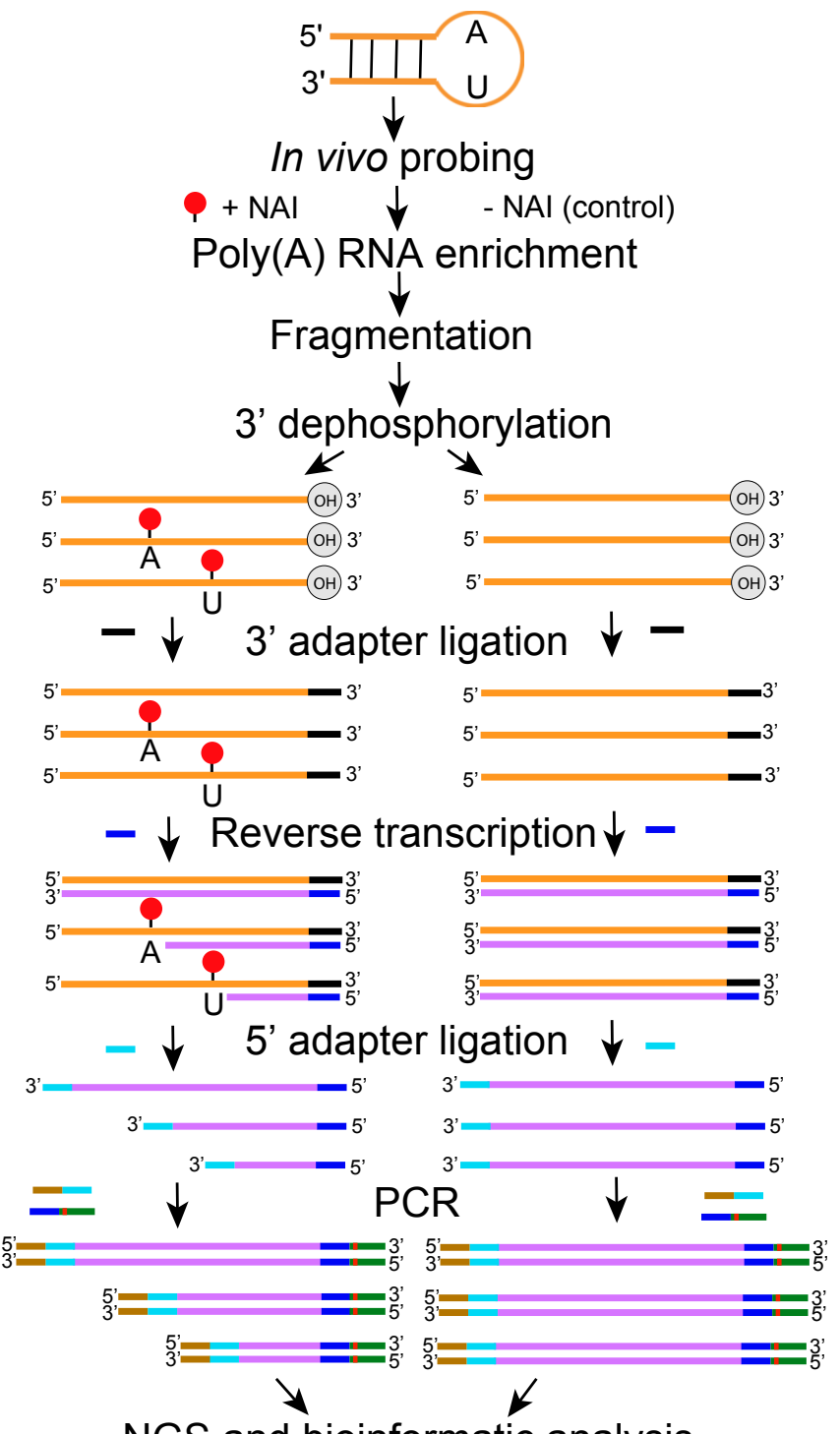

NGS and bioinformatic analysis

$\downarrow$

Structural reactivity sequence: ...(((((...))))).

Transcript folding:

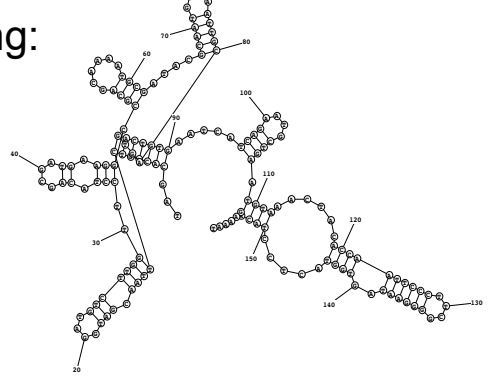

B 0000000000 0000000000 0000000000 0000000000 0000000000 0000000000 0000000000 0000000000 0000000000 0000000000

Total $=2669$

C

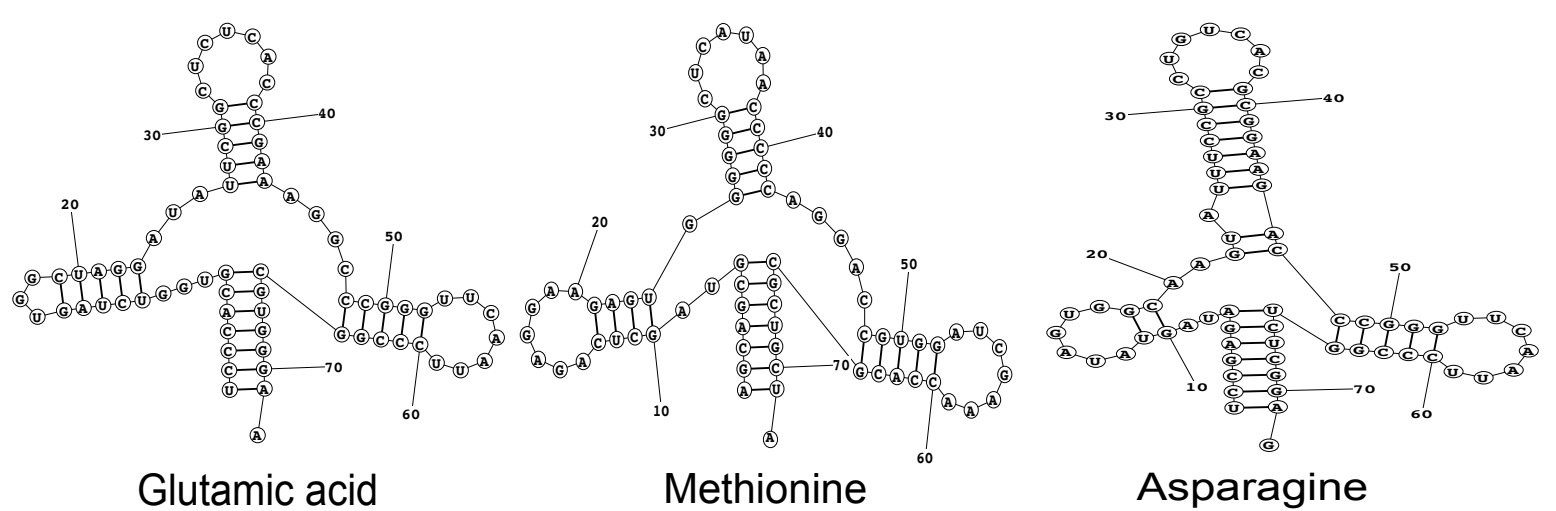

Glutamic acid

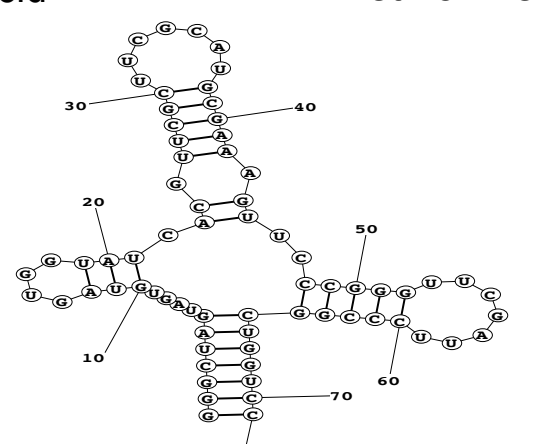

Alanine

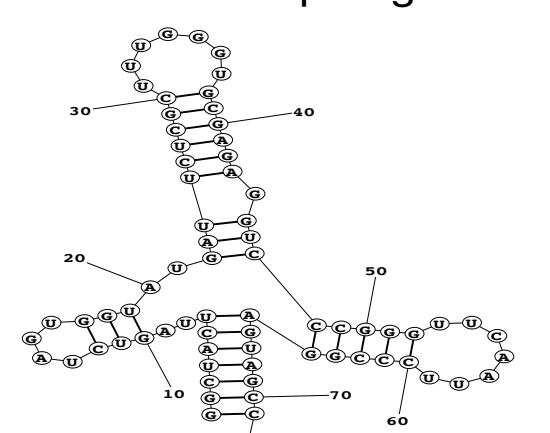

Proline uncharacterised non protein coding $(0.94 \%)$

$\square$ snoRNA $(1.31 \%) \square$ mito_rRNA $(1.16$
$\square \operatorname{rRNA}(0.45 \%) \square \operatorname{tRNA}(0.34 \%)$

$\square \operatorname{snRNA}(0.19 \%) \quad \square$ mito_mRNA $(0.11 \%)$ 

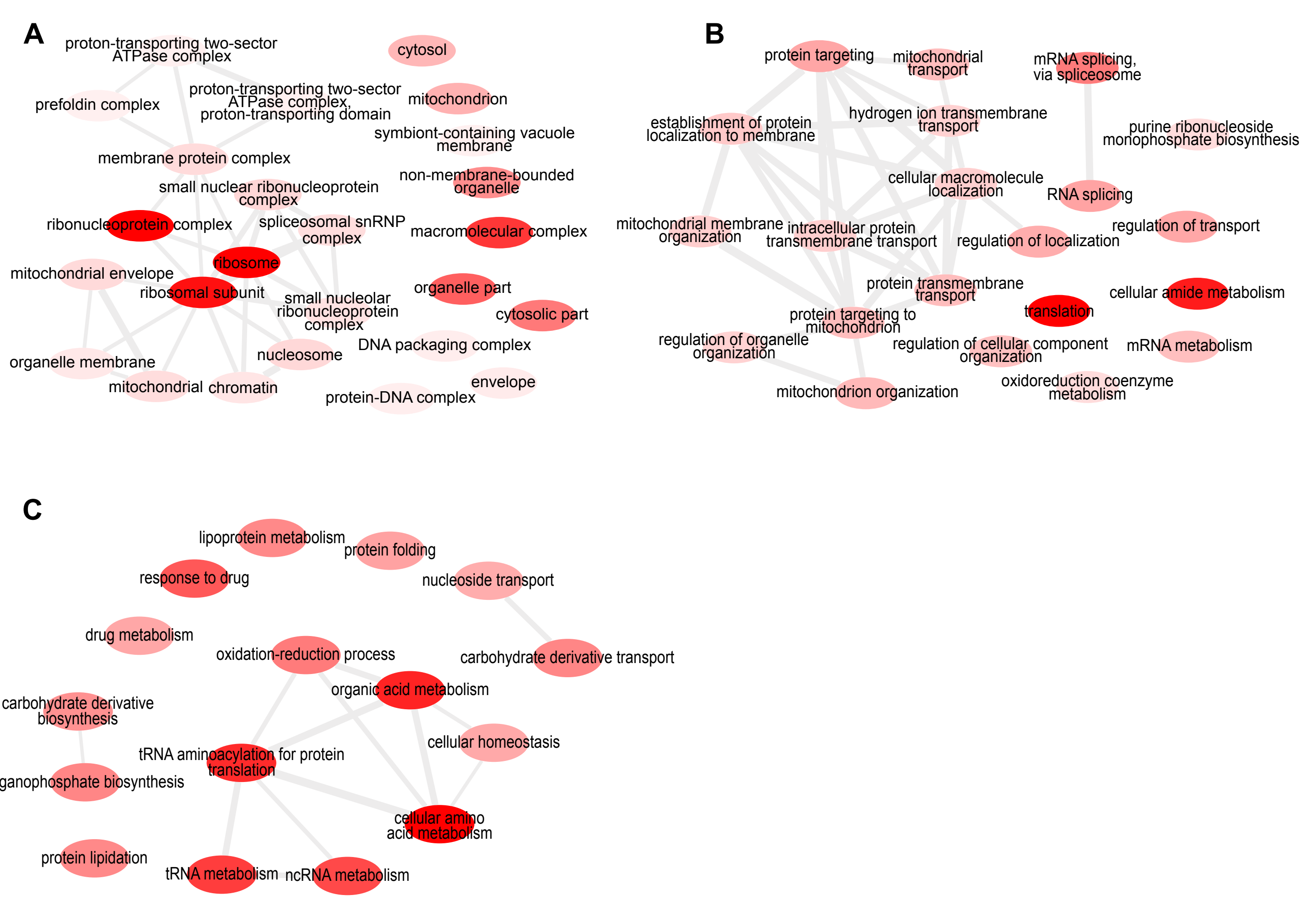


\section{A}

$$
10
$$

30

40

50

60

70

90

100

Sequence CACCCUUCUCGGCCUCUUGGCUAAGAUCAAUUUGUAGUACCUGUUCUUAUCAGCGUGAUAGCUGAUAUGGUCUCAAUAGAGGCCUUAUCAAUUUCACAAA

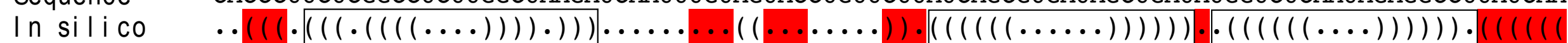

St r uct ur e-seq.

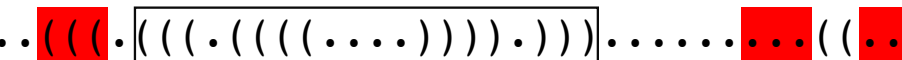

......

$\frac{(((((((\ldots \ldots)))))))}{150} \cdot((((((\ldots)))))) \cdot \ldots \ldots$

110

140

1

।

Sequence AAUUUUUGAUAGGGGAAAGGAUAAUUUGAAGCUUGCUUCUUAUAACCUUUCGCGCGUUGCCUUACCCUUGCACUAAAGGUUUGUACAGUGCACCCUUA In silico

St r uct ur e-seq

$\ldots \ldots()))())))((((((.((\cdot(. .((((\ldots)))) \ldots))) \cdot)))))((((\ldots(((\ldots(((\bullet((\ldots \ldots)))) \ldots))) \ldots)))) \ldots \ldots$

B
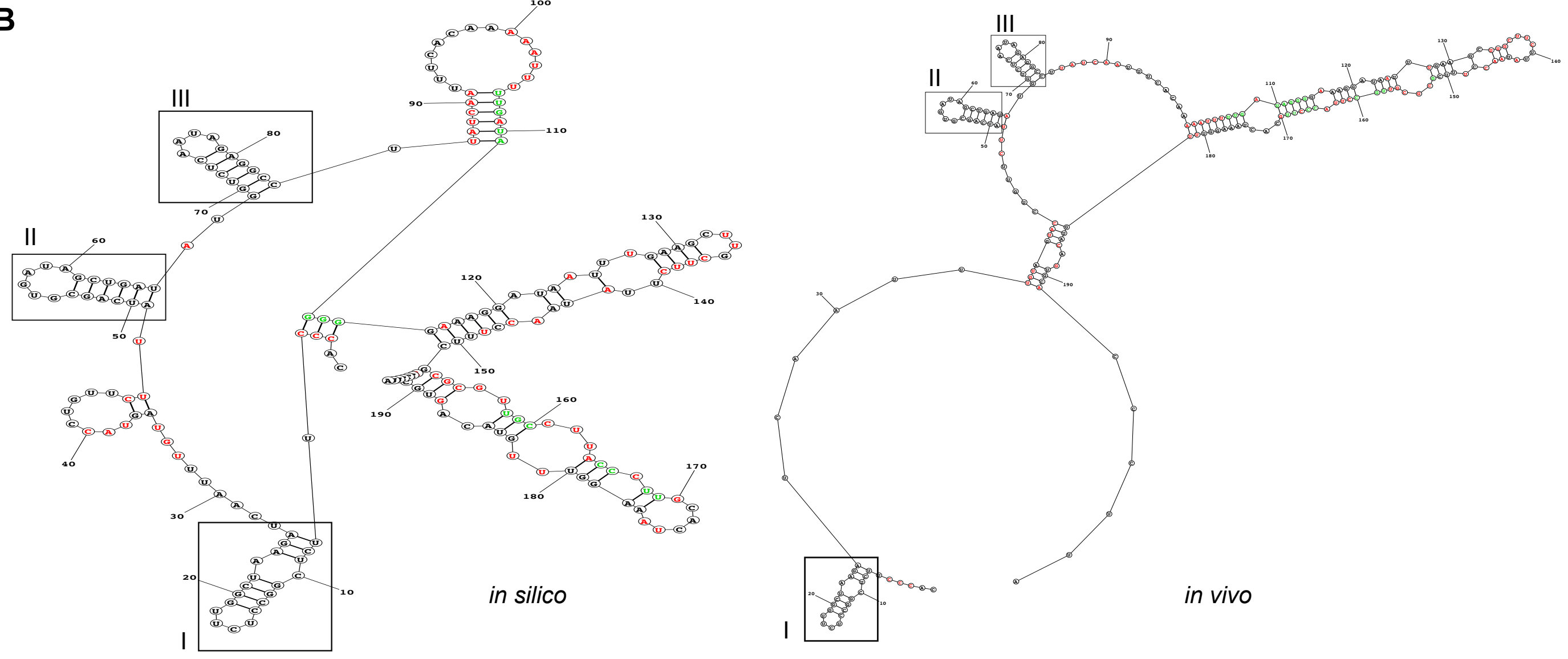

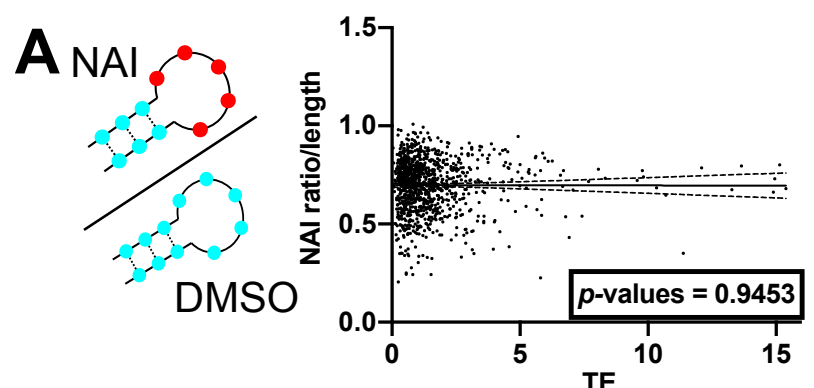

B

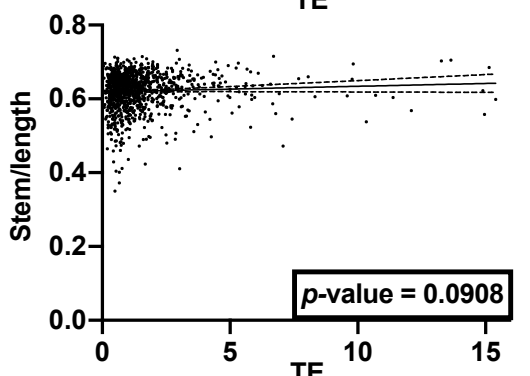

C

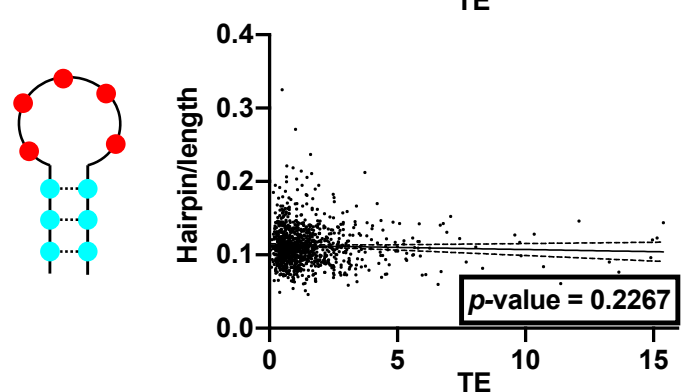

D

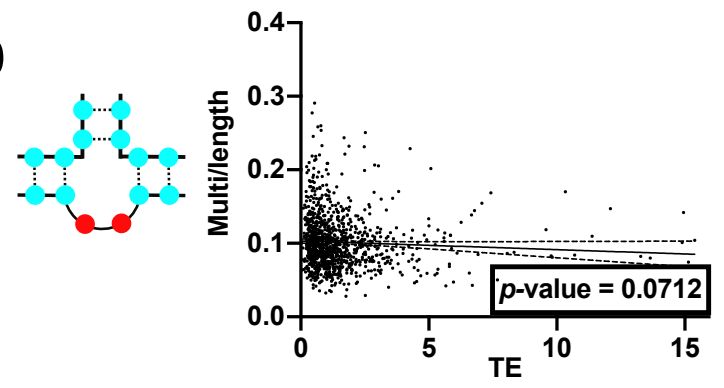

E

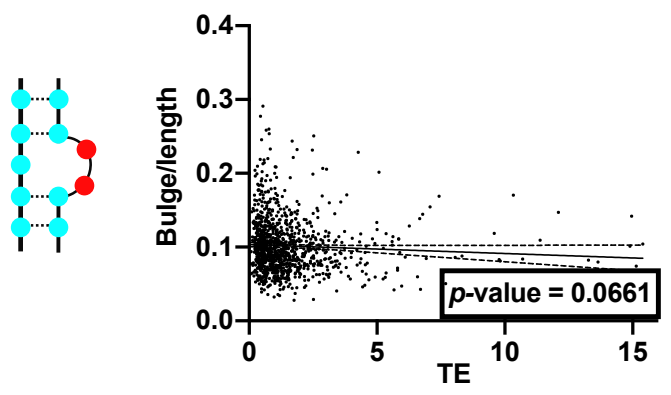

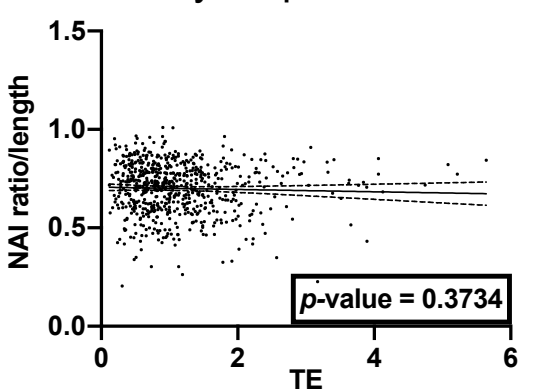
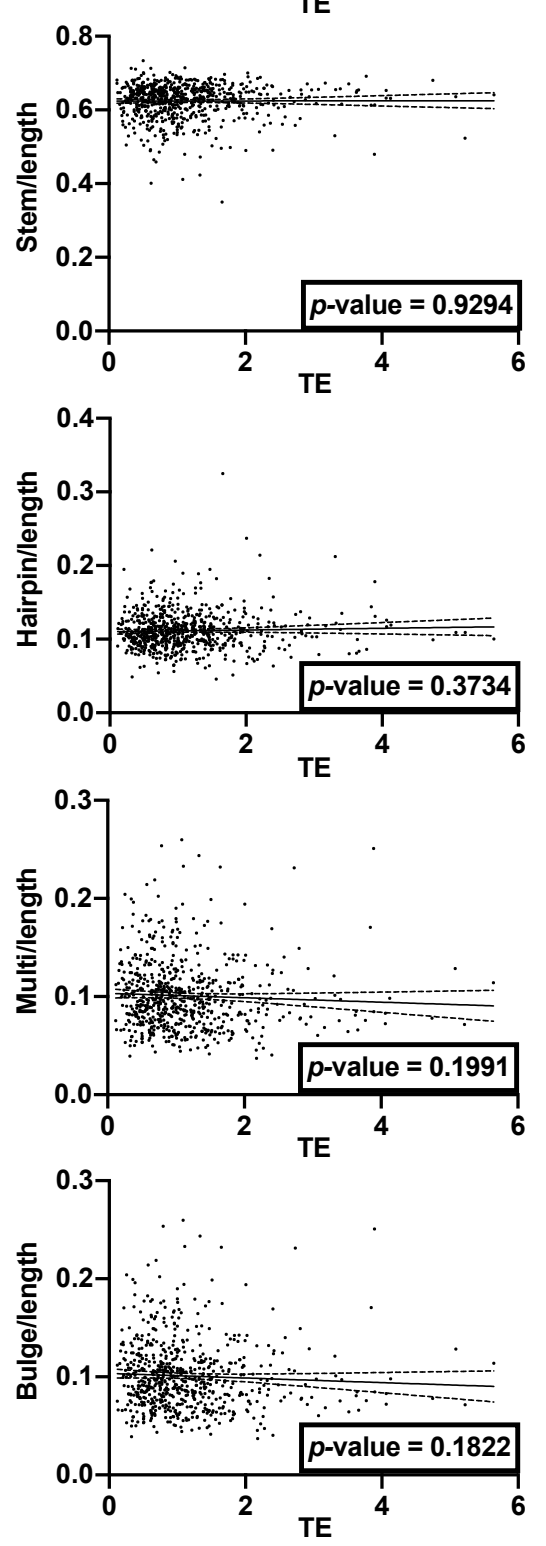
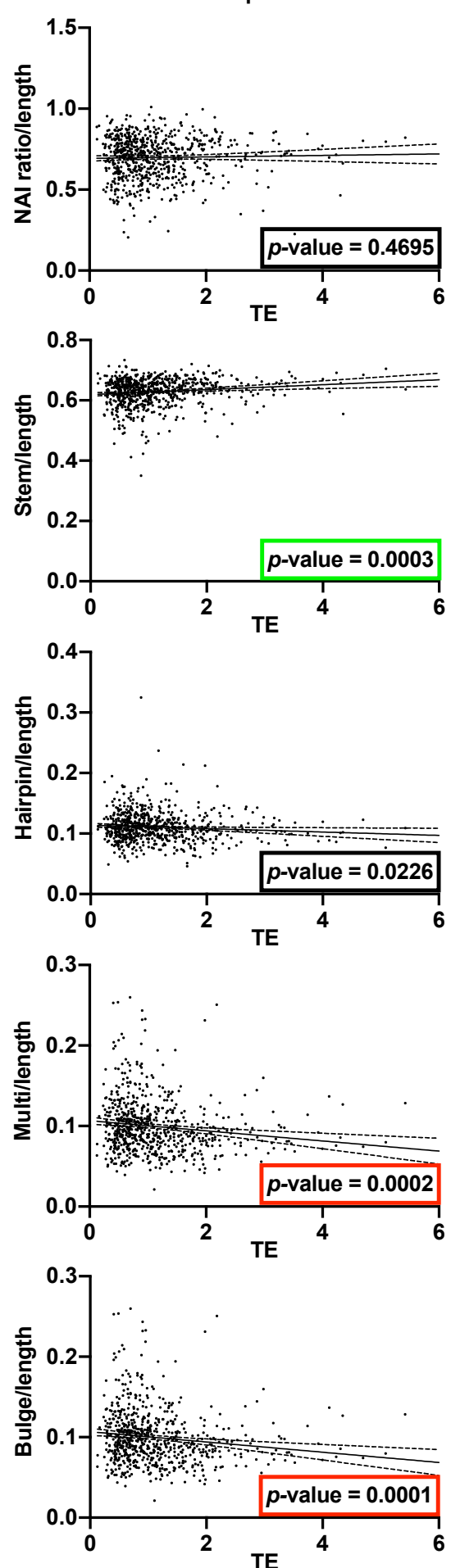
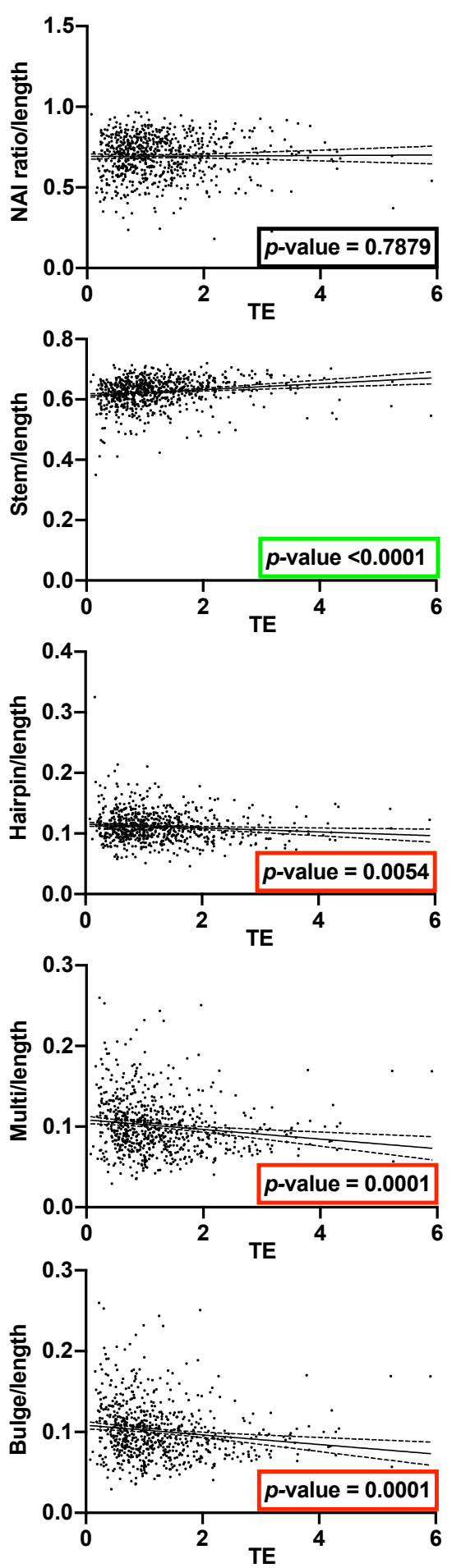
Rings
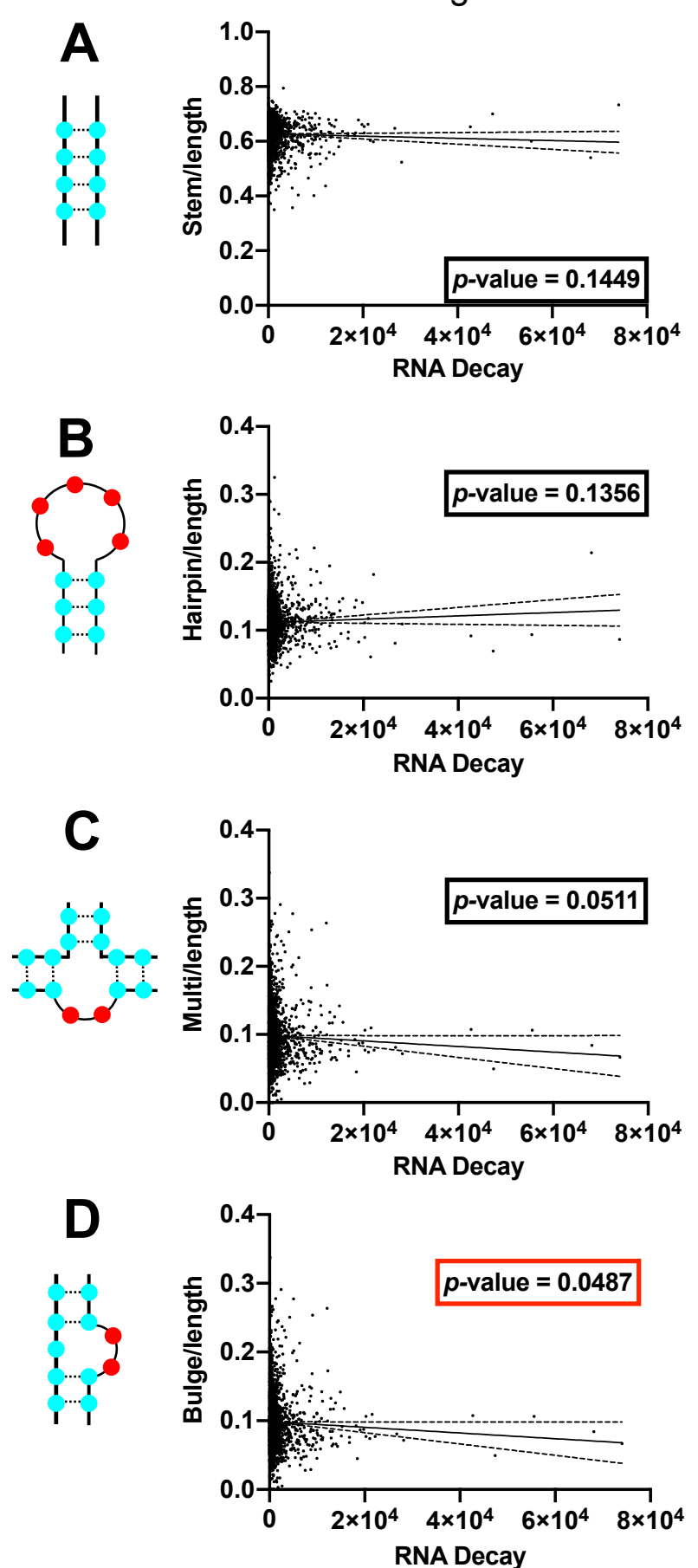

Early Trophozoites
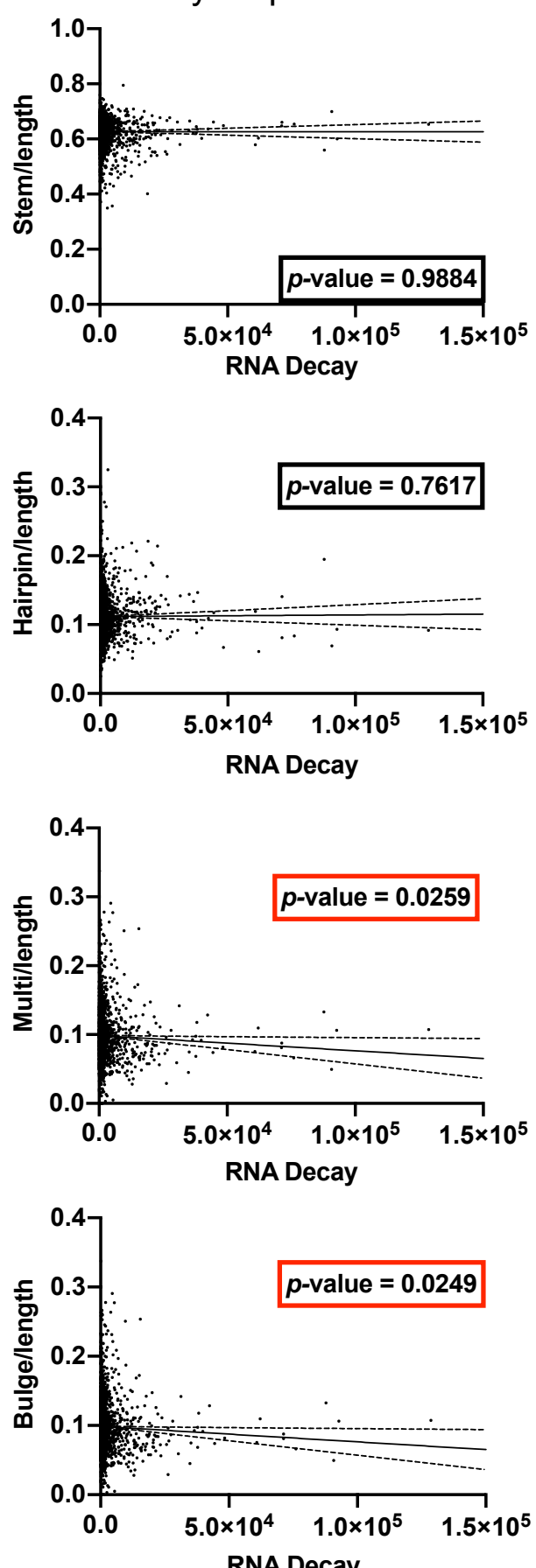

Late Trophozoites
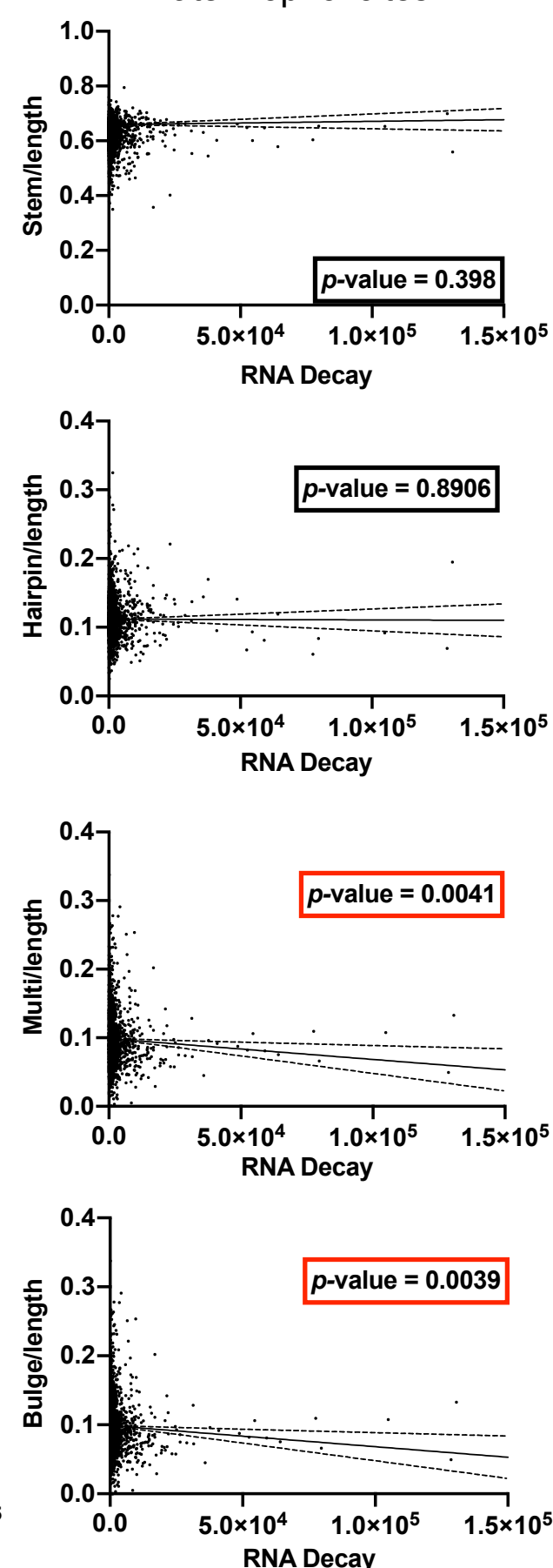

Schizonts
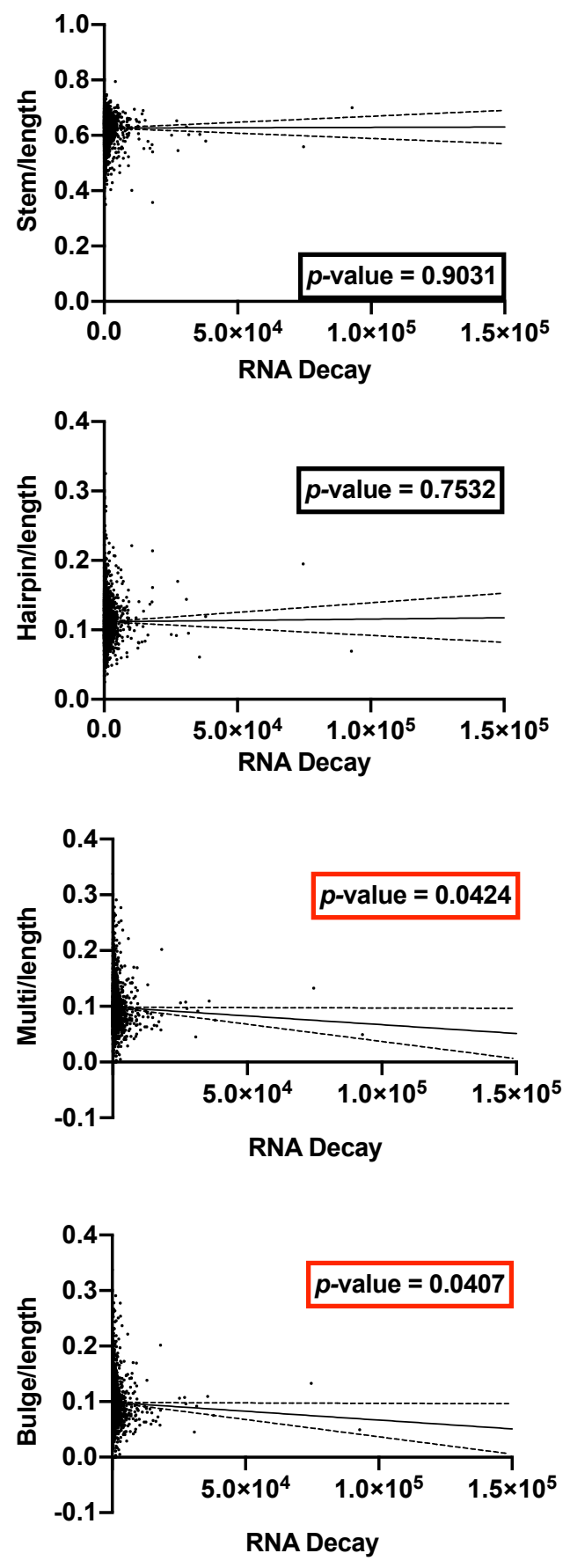